\title{
ON THE FUNCTORIALITY OF MARKED FAMILIES
}

\author{
PAOLO LELLA AND MARGHERITA ROGGERO
}

\begin{abstract}
The application of methods of computational algebra has recently introduced new tools for the study of Hilbert schemes. The key idea is to define flat families of ideals endowed with a scheme structure whose defining equations can be determined by algorithmic procedures. For this reason, several authors developed new methods, based on the combinatorial properties of Borel-fixed ideals, that allow associating to each ideal $J$ of this type a scheme $\mathbf{M f}_{J}$, called a $J$-marked scheme. In this paper, we provide a solid functorial foundation to marked schemes and show that the algorithmic procedures introduced in previous papers do not depend on the ring of coefficients. We prove that, for all strongly stable ideals $J$, the marked schemes $\mathbf{M f}_{J}$ can be embedded in a Hilbert scheme as locally closed subschemes, and that they are open under suitable conditions on $J$. Finally, we generalize Lederer's result about Gröbner strata of zero-dimensional ideals, proving that Gröbner strata of any ideals are locally closed subschemes of Hilbert schemes.
\end{abstract}

Introduction. This article aims to give a solid functorial foundation for the theory of marked schemes over a strongly stable ideal $J$ introduced in $[4,9]$. We describe the foundation in terms of representable functors and prove that these functors are represented by the schemes constructed in the aforementioned papers. Moreover, under mild additional hypotheses on $J$, these functors turn out to be subfunctors of a Hilbert functor. Equations defining the marked schemes can be effectively computed; therefore, these methods allow for effective computations on Hilbert schemes. In particular, if we only consider algebras and schemes over a field of characteristic 0 , marked schemes

2010 AMS Mathematics subject classification. Primary 13P99, $14 \mathrm{C} 05$.

Keywords and phrases. Hilbert scheme, marked family, Borel-fixed ideal, open subfunctor.

The first author was partially supported by GNSAGA of INdAM, by PRIN 20102011 "Geometria delle varietà algebriche," and by FIRB 2012 "Moduli spaces and Applications." The second author was supported by PRIN 2010-2011 "Geometria delle varietà algebriche."

Received by the editors on September 17, 2013, and in revised form on April 8, 2015. 
Mf $f_{J}$ with $J$ strongly stable provide, up to the action of the linear group, an open cover of the Hilbert scheme.

For a given monomial ideal $J$ in a polynomial ring $A\left[x_{0}, \ldots, x_{n}\right]$, we consider the collection of all ideals $I$ such that

$$
A\left[x_{0}, \ldots, x_{n}\right]=I \oplus\langle\mathcal{N}(J)\rangle,
$$

where $\mathcal{N}(J)$ denotes the set of monomials not contained in $J$. In the case where $A$ is a field and $J$ is strongly stable, this collection appears for the first time in [9], where it is called a $J$-marked family, and it is proved that it can be endowed with a structure of scheme (called a $J$-marked scheme) $[\mathbf{4}, \mathbf{9}]$.

All ideals $I$ of this collection share the same basis $\mathcal{N}(J)$ of the quotient algebra $A\left[x_{0}, \ldots, x_{n}\right] / I$; therefore, they define subschemes in $\operatorname{Proj} A\left[x_{0}, \ldots, x_{n}\right]$ with the same Hilbert polynomial. These same properties hold for Gröbner strata, which are schemes parametrizing homogeneous ideals having a fixed monomial ideal as their initial ideal with respect to a given term ordering. However, we emphasize that marked schemes and Gröbner strata are not the same objects. Indeed, in general, a $J$-marked scheme strictly contains the Gröbner stratum with initial ideal $J$ with respect to a fixed term ordering (or even the union of all Gröbner strata with initial ideal $J$ ).

The use of Gröbner strata in the study of Hilbert schemes is very natural and has been discussed since [3, 7]. Indeed, the ideals of a Gröbner stratum define points on the same Hilbert scheme, and Gröbner strata cover set-theoretically the Hilbert scheme. Thus, several authors addressed the question whether a Gröbner stratum can be equipped by a scheme structure and, if so, how this scheme is embedded in the Hilbert scheme.

Notari and Spreafico [24] proved that every Gröbner stratum (considering the reverse lexicographic order) is a locally closed subscheme of the support of the Hilbert scheme. Lederer [19] obtained a stronger result in the case of Hilbert schemes of points; working in the affine framework, he proved that Gröbner strata are locally closed subschemes of the Hilbert scheme. In [22], the authors of the present paper found suitable conditions on the monomial ideal $J$ and on the term ordering that are sufficient to ensure that the Gröbner stratum is an open subscheme of the Hilbert scheme. 
Nevertheless, Gröbner strata are in general not sufficient for obtaining an open cover of the Hilbert scheme (see $[\mathbf{9}, \mathbf{2 2}])$ although we can obtain such an open cover using marked schemes and exploiting the action of the general linear group on the Hilbert scheme. Furthermore, equations defining a $J$-marked scheme can be computed by some algorithmic procedures developed in $[4, \mathbf{5}, \mathbf{9}]$. The key point is a procedure of polynomial reduction, similar to the one for Gröbner bases, but that does not need a term ordering (see Definition 2.8).

In this paper, we prove that the procedure of reduction is also "natural." Indeed, the reduction works independently of the ring $A$ of coefficients of the polynomial ring, so that the schemes introduced in $[4,5,9]$ correctly describe the scheme structure of the Hilbert scheme (Theorem 3.4 and Corollary 4.3).

In the classical construction of the Hilbert scheme, every point is associated to the homogeneous piece of a, sufficiently large, degree $r$ of the ideal defining the corresponding scheme. At first sight, one could be tempted to consider a marked scheme over ideals truncated in the same (large) degree. However, explicit computations of these marked schemes turn out to be, in general, out of reach due to the huge number of variables required. Since the number of variables depends on the degree of the truncation, we develop the theory of marked functors in a wider generality, considering marked functors over ideals truncated in any degree. In this way, we can find marked schemes that correctly describe the local structure of the Hilbert scheme but that are far easier to compute (Theorem 3.4).

Finally, we discuss the relation between marked schemes and Gröbner strata, also introducing a representable functor whose representing scheme is in fact a Gröbner stratum. For constant Hilbert polynomials, the Gröbner strata we define in the projective case coincide with those introduced by Lederer in the affine case. In this paper, we generalize the Lederer result to Hilbert polynomials of any degree, proving that Gröbner strata are closed subschemes of marked schemes, and so, locally closed subschemes of the Hilbert scheme (Theorem 5.3).

In the last section, we discuss in detail the case of a strongly stable ideal defining a zero-dimensional subscheme of $\mathbb{P}^{3}$ of degree 7 . We explicitly compute the equations of marked schemes and Gröbner 
strata of different truncations, showing the relations among them and exhibiting several phenomena described in the paper.

1. Marked bases. In this section, we recall the main definitions concerning sets of polynomials marked over a monomial ideal $J$, and we describe some properties of an ideal generated by such a set, assuming that $J$ is strongly stable.

First, let us set some notation. Throughout the paper, we will consider Noetherian rings. We will denote by $\mathbb{Z}[\mathbf{x}]$ the polynomial ring $\mathbb{Z}\left[x_{0}, \ldots, x_{n}\right]$ and by $\mathbb{P}_{\mathbb{Z}}^{n}$ the projective space Proj $\mathbb{Z}[\mathbf{x}]$. For any ring $A$, $A[\mathbf{x}]$ will denote the polynomial ring $A \otimes_{\mathbb{Z}} \mathbb{Z}[\mathbf{x}]$ in $n+1$ variables with coefficients in $A$, and $\mathbb{P}_{A}^{n}$ will be the scheme

$$
\operatorname{Proj} A[\mathbf{x}]=\mathbb{P}_{\mathbb{Z}}^{n} \times_{\operatorname{Spec} \mathbb{Z}} \operatorname{Spec} A .
$$

For every integer $s$, we denote by $A[\mathbf{x}]_{s}$ the graded component of degree $s$, and we set

$$
D_{s}:=D \cap A[\mathbf{x}]_{s} \text { for every } D \subseteq A[\mathbf{x}] .
$$

We denote monomials in multi-index notation. For any element $\alpha=\left(\alpha_{0}, \ldots, \alpha_{n}\right) \in \mathbb{N}^{n+1}, x^{\alpha}$ will be the monomial $x_{0}^{\alpha_{0}} \cdots x_{n}^{\alpha_{n}}$ and $|\alpha|$ will be its degree. Given a set of homogeneous polynomials $H$ in $A[\mathbf{x}]$, in order to emphasize dependence on the coefficient ring $A$, we write ${ }_{A}\langle H\rangle$ for the $A$-module generated by $H$ and ${ }_{A}(H)$ for the ideal in $A[\mathbf{x}]$ generated by $H$. We will omit this subscript when no ambiguity can arise, for instance, when only one $\operatorname{ring} A$ is involved.

If $J$ is a monomial ideal in $A[\mathbf{x}]$, then $\mathcal{B}_{J}$ is its minimal set of generators and $\mathcal{N}(J)$ is the set of monomials not contained in $J$.

Remark 1.1. A monomial ideal is determined by the set of monomials it contains. In the following, by abuse of notation, we will use the same letter to denote all monomial ideals having the same set of monomials, even in polynomial rings with different rings of coefficients. More formally, if $J$ is a monomial ideal in $\mathbb{Z}[\mathbf{x}]$, we will denote, by the same symbol $J$, all the ideals $J \otimes_{\mathbb{Z}} A$.

Throughout the paper, we assume the variables are ordered as $x_{0}<\cdots<x_{n}$. For any monomial $x^{\alpha}$, we denote by $\min x^{\alpha}$ the 
smallest variable, or equivalently its index, dividing $x^{\alpha}$ and by $\max x^{\alpha}$ the greatest variable, or its index, dividing the monomial.

Definition 1.2. An ideal $J \subseteq A[\mathrm{x}]$ is said to be strongly stable if

(i) $J$ is a monomial ideal;

(ii) if $x^{\alpha} \in J$, then $\left(x_{i} / x_{j}\right) x^{\alpha} \in J$, for all $x_{j} \mid x^{\alpha}$ and $x_{i}>x_{j}$.

These ideals are extensively studied in commutative algebra and widely used in algebraic geometry since they are related to the Borelfixed ideals [14]. Indeed, every strongly stable ideal is Borel-fixed, whereas in general, a Borel-fixed ideal does not need to be strongly stable. The two notions coincide in polynomial rings with coefficients in a field of characteristic 0. Borel-fixed ideals are involved in some of the most important general results on Hilbert schemes, as for instance, the proof of its connectedness given by Hartshorne [17].

Combinatorial properties of strongly stable ideals have been successfully used for designing algorithms inspired by the theory of Gröbner bases but not requiring a term ordering. The role of the term ordering, a total ordering on the set of monomials, is played by a partial order called the Borel ordering, given as the transitive closure of the relation

$$
x^{\alpha}>_{B} x^{\beta} \Longleftrightarrow x_{i} x^{\alpha}=x_{j} x^{\beta} \quad \text { and } \quad x_{i}<x_{j} .
$$

Moving from this order, it is possible to define reduction procedures which turn out to be Noetherian. A detailed description of these techniques is contained in the papers $[\mathbf{4}, \mathbf{5}, \mathbf{9}]$. We will now recall some of the main properties needed in the next section.

Definition 1.3. For a polynomial $f \in A[\mathbf{x}]$, its support, denoted by $\operatorname{Supp}(f)$, is the set of monomials appearing in $f$ with a non-zero coefficient. We refer to the set of non-zero coefficients of $f$ as $\mathbf{x}$ coefficients of $f$. A monic marked polynomial is a polynomial $f \in A[\mathbf{x}]$ with a specified monomial $\operatorname{Ht}(f)$ of its support, with coefficient $1_{A}$. We call $\operatorname{Ht}(f)$ the head term of $f$, and we call $\mathrm{T}(f):=\operatorname{Ht}(f)-f$ the tail of $f$ (so that $f=\operatorname{Ht}(f)-\mathrm{T}(f)$ ). Throughout the paper, we describe marked polynomials adding as a subscript the multi-index corresponding to the head term, i.e., we write $f_{\alpha}$ meaning that $\operatorname{Ht}\left(f_{\alpha}\right)=x^{\alpha}$. 
Definition 1.4. Let $J \subseteq A[\mathbf{x}]$ be a strongly stable ideal, and let $\mathcal{B}_{J}$ be the minimal set of generators of $J$. We call a $J$-marked set a set of monic marked polynomials

$$
\left\{f_{\alpha}:=x^{\alpha}-\sum_{x^{\beta} \in \mathcal{N}(J)_{|\alpha|}} c_{\alpha \beta} x^{\beta} \in A[\mathbf{x}] \mid x^{\alpha} \in \mathcal{B}_{J}\right\},
$$

where $\operatorname{Ht}\left(f_{\alpha}\right)=x^{\alpha}$ and $c_{\alpha \beta} \in A$. A $J$-marked set $F_{J}$ is called a $J$ marked basis if $A[\mathbf{x}]={ }_{A}\left(F_{J}\right) \oplus{ }_{A}\langle\mathcal{N}(J)\rangle$, i.e., the monomials of $\mathcal{N}(J)$ freely generate $A[\mathbf{x}] / A_{A}\left(F_{J}\right)$.

We emphasize that the assumption of the head term to be monic is significant only if the ring of coefficients $A$ is not a field. Indeed, if $A$ is a field (as in $[4,9]$ ), a set of marked polynomials can always be modified in a set of monic marked polynomials.

If $\left(F_{J}\right)$ is a $J$-marked basis, then the scheme Proj $A[\mathbf{x}] /\left(F_{J}\right)$ is $A$-flat because the $A$-module $A[\mathbf{x}] / A_{A}\left(F_{J}\right)$ is free. The ideal ${ }_{A}\left(F_{J}\right)$ generated by a $J$-marked basis $F_{J}$ has the same Hilbert polynomial as the monomial ideal $J$, so that $J$ and ${ }_{A}\left(F_{J}\right)$ define schemes corresponding to closed points of the same Hilbert scheme. Therefore, it is interesting to find theoretical conditions and effective procedures in order to state whether a marked set is a marked basis.

Proposition 1.5 ([4, Lemma 1.2], [11, Lemma 1.1]). Let $J$ be a strongly stable ideal.

(i) Each monomial $x^{\alpha}$ can be uniquely written as a product $x^{\gamma} x^{\delta}$ with $x^{\gamma} \in \mathcal{B}_{J}$ and $\min x^{\gamma} \geq \max x^{\delta}$. Therefore, $x^{\delta}<_{\text {Lex }} x^{\eta}$ for every monomial $x^{\eta}$ such that $x^{\eta} \mid x^{\alpha}$ and $x^{\alpha-\eta} \notin J$. We will write $x^{\alpha}=x^{\gamma} *_{J} x^{\delta}$ to refer to this unique decomposition.

(ii) Consider $x^{\alpha} \in J \backslash \mathcal{B}_{J}$, and let $x_{j}=\min x^{\alpha}$. Then, $x^{\alpha} / x_{j}$ is contained in $J$.

(iii) Let $x^{\beta}$ be a monomial not contained in J. If $x^{\delta} x^{\beta} \in J$, then either $x^{\delta} x^{\beta} \in \mathcal{B}_{J}$ or $x^{\delta} x^{\beta}=x^{\alpha} *_{J} x^{\delta^{\prime}}$ with $x^{\alpha} \in \mathcal{B}_{J}$ and $x^{\delta}>_{\text {Lex }} x^{\delta^{\prime}}$. In particular, if $x_{i} x^{\beta} \in J$, then either $x_{i} x^{\beta} \in \mathcal{B}_{J}$ or $x_{i}>\min x^{\beta}$.

Definition 1.6. Let $J$ be a strongly stable ideal, and let $I$ be the ideal generated by a $J$-marked set $F_{J}$ in $A[\mathbf{x}]$. We consider the following sets of polynomials: 
- $F_{J}^{(s)}:=\left\{x^{\delta} f_{\alpha} \mid \operatorname{deg}\left(x^{\delta} f_{\alpha}\right)=s, f_{\alpha} \in F_{J}, \min x^{\alpha} \geq \max x^{\delta}\right\}$;

- $\widehat{F}_{J}^{(s)}:=\left\{x^{\delta} f_{\alpha} \mid \operatorname{deg}\left(x^{\delta} f_{\alpha}\right)=s, f_{\alpha} \in F_{J}, \min x^{\alpha}<\max x^{\delta}\right\}$;

- $S F_{J}^{(s)}:=\left\{x^{\delta} f_{\beta}-x^{\gamma} f_{\alpha} \mid x^{\delta} f_{\beta} \in \widehat{F}_{J}^{(s)}, x^{\gamma} f_{\alpha} \in F_{J}^{(s)}, x^{\delta} x^{\beta}=x^{\gamma} x^{\alpha}\right\}$;

- $\mathcal{N}(J, I):=I \cap{ }_{A}\langle\mathcal{N}(J)\rangle$.

Throughout the paper, we use the convention that, when multiplying a marked polynomial $f$ by a monomial $x^{\delta}$, we have $\operatorname{Ht}\left(x^{\delta} f\right)=x^{\delta} \operatorname{Ht}(f)$. Therefore, for each monomial $x^{\gamma} \in J_{s}$, there is a unique polynomial in $F_{J}^{(s)}$ with head term $x^{\gamma}$.

Theorem 1.7. Let $J$ be a strongly stable ideal and $I \subseteq A[\mathbf{x}]$ the ideal generated by a $J$-marked set $F_{J}$. For every $s$,

(i) $I_{s}=\left\langle F_{J}^{(s)}\right\rangle+\left\langle\widehat{F}_{J}^{(s)}\right\rangle=\left\langle F_{J}^{(s)}\right\rangle+\left\langle S F_{J}^{(s)}\right\rangle$;

(ii) $A[\mathbf{x}]_{s}=\left\langle F_{J}^{(s)}\right\rangle \oplus\left\langle\mathcal{N}(J)_{s}\right\rangle$;

(iii) the $A$-module $\left\langle F_{J}^{(s)}\right\rangle$ is free of rank equal to $\mathrm{rk} J_{s}$ and is generated by a unique $\left(J_{s}\right)$-marked set $\widetilde{F}_{J}^{(s)}$;

(iv) $I_{s}=\left\langle F_{J}^{(s)}\right\rangle \oplus \mathcal{N}(J, I)_{s}=\left\langle\widetilde{F}_{J}^{(s)}\right\rangle \oplus \mathcal{N}(J, I)_{s}$.

Moreover, the following are equivalent:

(v) $F_{J}$ is a $J$-marked basis;

(vi) for all $s, I_{s}=\left\langle F_{J}^{(s)}\right\rangle$;

(vii) for all $s,\left\langle S F_{J}^{(s)}\right\rangle \subseteq\left\langle F_{J}^{(s)}\right\rangle$;

(viii) $\mathcal{N}(J, I)=0$.

Proof.

(i) Straightforward from the definition of the homogeneous piece of a given degree of an ideal.

(ii) We start by proving that there are no non-zero polynomials in the intersection $\left\langle F_{J}^{(s)}\right\rangle \cap\left\langle\mathcal{N}(J)_{s}\right\rangle$. Let us consider

$$
h:=\sum_{i} b_{i} x^{\delta_{i}} f_{\alpha_{i}}
$$

where $x^{\delta_{i}} f_{\alpha_{i}}$ are distinct elements of $F_{J}^{(s)}$ and $b_{i} \in A \backslash\{0\}$. Assume that the polynomials $x^{\delta_{i}} f_{\alpha_{i}}$ are indexed so that $x^{\delta_{1}} \geq_{\text {Lex }} x^{\delta_{2}} \geq_{\text {Lex }} \cdots$. Then $b_{1}$ also turns out to be the coefficient of the monomial $x^{\delta_{1}} x^{\alpha_{1}}$ in $h$. Indeed, $x^{\alpha_{1}} x^{\delta_{1}}$ does not appear either as a head term or in the support 
of the tail of a summand $x^{\delta_{i}} f_{\alpha_{i}}$ of $h$ with $i>1$. The monomial cannot be the head term of $x^{\delta_{i}} f_{\alpha_{i}}$, since the head terms in $F_{J}^{(s)}$ (and so in the summands of $h$ ) are all different. Moreover, $x^{\alpha_{1}} x^{\delta_{1}}$ cannot appear in $T\left(x^{\delta_{i}} f_{\alpha_{i}}\right)$ with $i>1$, since it has the unique decomposition $x^{\alpha_{1}} *_{J} x^{\delta_{1}}$, while every monomial $x^{\delta_{i}} x^{\beta} \in T\left(x^{\delta_{i}} f_{\alpha_{i}}\right) \cap J$ has decomposition $x^{\alpha} *_{J} x^{\eta}$ with $x^{\eta}<_{\text {Lex }} x^{\delta_{i}}<_{\text {Lex }} x^{\delta_{1}}$ by Proposition 1.5 (iii) (note that, by definition, $\left.x^{\beta} \in \operatorname{Supp}\left(T\left(f_{\alpha_{1}}\right)\right) \subseteq \mathcal{N}(J)\right)$. Therefore, no non-zero polynomials in $\left\langle F_{J}^{(s)}\right\rangle$ are contained in $\left\langle\mathcal{N}(J)_{s}\right\rangle$.

To conclude the proof, we show that every monomial $x^{\beta}$ of degree $s$ is contained in the direct $\operatorname{sum}\left\langle F_{J}^{(s)}\right\rangle \oplus\left\langle\mathcal{N}(J)_{s}\right\rangle$. If $x^{\beta} \in \mathcal{N}(J)_{s}$, there is nothing to prove. Now assume that there exists some monomial in $J_{s}$ not contained in $\left\langle F_{J}^{(s)}\right\rangle \oplus\left\langle\mathcal{N}(J)_{s}\right\rangle$. Among them, choose $x^{\beta}$ such that, in the unique decomposition $x^{\beta}=x^{\alpha} *_{J} x^{\delta}$, monomial $x^{\delta}$ is minimum with respect to the Lex ordering. Since $x^{\beta}=x^{\delta} f_{\alpha}+\mathrm{T}\left(x^{\delta} f_{\alpha}\right)$, the support of $\mathrm{T}\left(x^{\delta} f_{\alpha}\right)$ cannot be contained in $\mathcal{N}(J)_{s}$, i.e., there exists $x^{\eta} \in \operatorname{Supp}\left(T\left(f_{\alpha}\right)\right)$ such that $x^{\eta} x^{\delta} \in J$. By Proposition 1.5 (iii), we have the decomposition $x^{\eta} x^{\delta}=x^{\alpha^{\prime}} *_{J} x^{\delta^{\prime}}$ with $x^{\delta^{\prime}}<_{\text {Lex }} x^{\delta}$ against the assumption of minimality on $x^{\delta}$.

(iii) By (ii), we have the short exact sequence

$$
0 \longrightarrow\left\langle F_{J}^{(s)}\right\rangle \hookrightarrow A[\mathbf{x}]_{s} \stackrel{\pi}{\longrightarrow}\left\langle\mathcal{N}(J)_{s}\right\rangle \longrightarrow 0 .
$$

For each $x^{\alpha}$ in $J_{s}$, we compute the image

$$
\pi\left(x^{\alpha}\right)=\sum_{x^{\beta} \in \mathcal{N}(J)_{s}} a_{\alpha \beta} x^{\beta},
$$

and consider the set

$$
\widetilde{F}_{J}^{(s)}:=\left\{\widetilde{f}_{\alpha}:=x^{\alpha}-\sum_{x^{\beta} \in \mathcal{N}(J)_{s}} a_{\alpha \beta} x^{\beta} \mid x^{\alpha} \in J_{s}\right\} \subseteq \operatorname{ker} \pi=\left\langle F_{J}^{(s)}\right\rangle .
$$

Let $J^{\prime}:=\left(J_{s}\right)$. By construction, the set $\widetilde{F}_{J}^{(s)}$ is a $J^{\prime}$-marked set with $\operatorname{Ht}\left(\widetilde{f}_{\alpha}\right)=x^{\alpha}$. Applying (ii) to this $J^{\prime}$-marked set, we have $\left\langle\widetilde{F}_{J}^{(s)}\right\rangle \oplus\left\langle\mathcal{N}\left(J^{\prime}\right)_{s}\right\rangle=A[\mathbf{x}]_{s}$. Finally, since the $A$-module generated by $\widetilde{F}_{J}^{(s)}$ is contained in $\left\langle F_{J}^{(s)}\right\rangle$ and $\mathcal{N}(J)_{s}=\mathcal{N}\left(J^{\prime}\right)_{s}$, the modules $\left\langle\widetilde{F}_{J}^{(s)}\right\rangle$ and $\left\langle F_{J}^{(s)}\right\rangle$ coincide. Note that $\widetilde{F}_{J}^{(s)}$ is marked on the monomial ideal $J^{\prime}$ generated by $J_{s}$ but does not need to be a $J_{\geq s}$-marked set, since $J_{\geq s}$ may have minimal generators of degree $>s$. 
(iv) By (i) and (iii), we have $I_{s}=\left\langle\widetilde{F}_{J}^{(s)}\right\rangle+\left\langle S F_{J}^{(s)}\right\rangle$. Since $\left\langle\widetilde{F}_{J}^{(s)}\right\rangle \cap$ $\left\langle\mathcal{N}(J)_{s}\right\rangle=\{0\}$, the module $\mathcal{N}\left(J, F_{J}\right)_{s}$ can be determined starting from the generators of $\left\langle S F_{J}^{(s)}\right\rangle$ and replacing each monomial $x^{\beta} \in J_{s}$ appearing in some polynomial of $S F_{J}^{(s)}$ with the tail $\mathrm{T}\left(\widetilde{f}_{\beta}\right)$ of the polynomial $\widetilde{f}_{\beta} \in \widetilde{F}_{J}^{(s)}$ with $\operatorname{Ht}\left(\widetilde{f}_{\beta}\right)=x^{\beta}$. The result of this procedure is a set of polynomials contained both in $I_{s}$ and $\left\langle\mathcal{N}(J)_{s}\right\rangle$. The sum of $\mathcal{N}(J, I)_{s}$ and $\left\langle F_{J}^{(s)}\right\rangle$ is direct by (ii) and (iii).

The equivalences (v) $\Leftrightarrow$ (vi) $\Leftrightarrow$ (vii) $\Leftrightarrow$ (viii) follow directly from the first part of the theorem. In fact, these properties are a rephrasing of the definition of $J$-marked basis.

We emphasize that the above result does not hold in general for a monomial ideal $J$ which is not strongly stable, as shown by the following example.

Example 1.8. Consider $J=\left(x_{2}^{2}, x_{1}^{2}\right) \subseteq \mathbb{Z}\left[x_{0}, x_{1}, x_{2}\right]$, and let $I$ be the ideal generated by the $J$-marked set $F_{J}=\left\{f_{002}=x_{2}^{2}+x_{2} x_{1}, f_{020}=\right.$ $\left.x_{1}^{2}+x_{2} x_{1}\right\}$. An easy computation shows that $I_{3}$ is freely generated by $F_{J}^{(3)}$, but $\left|F_{J}^{(3)}\right|=\operatorname{rk} I_{3}=5<6=\operatorname{rk} J_{3}$, and $I_{3}$ does not contain any $\left(J_{3}\right)$-marked set $\widetilde{F}_{J}^{(3)}$.

Example 1.9. Consider the strongly stable ideal $J=\left(x_{2}^{2}, x_{2} x_{1}, x_{1}^{3}\right) \subseteq$ $\mathbb{Z}\left[x_{0}, x_{1}, x_{2}\right]$ and any $J$-marked set $F_{J}=\left\{f_{002}, f_{011}, f_{030}\right\}$ over a ring $A$. Let us compute the sets of polynomials $F_{J}^{(s)}, \widehat{F}_{J}^{(s)}$ and $S F_{J}^{(s)}$ discussed in Theorem 1.7 for $s=2,3,4$.

$$
\begin{aligned}
& (s=2) \quad F_{J}^{(2)}=\left\{f_{002}, f_{011}\right\}, \quad \widehat{F}_{J}^{(2)}=\emptyset, \quad S F_{J}^{(2)}=\emptyset, \\
& (s=3) \quad F_{J}^{(3)}=\left\{x_{2} f_{002}, x_{1} f_{002}, x_{0} f_{002}, x_{1} f_{011}, x_{0} f_{011}, f_{030}\right\}, \\
& \widehat{F}_{J}^{(3)}=\left\{x_{2} f_{011}\right\}, \quad S F_{J}^{(3)}=\left\{x_{2} f_{011}-x_{1} f_{002}\right\}, \\
& (s=4) \quad F_{J}^{(4)}=\left\{\begin{array}{l}
x_{2}^{2} f_{002}, x_{2} x_{1} f_{002}, x_{2} x_{0} f_{002}, x_{1}^{2} f_{002}, x_{1} x_{0} f_{002}, \\
x_{0}^{2} f_{002}, x_{1}^{2} f_{011}, x_{1} x_{0} f_{011}, x_{0}^{2} f_{011}, x_{1} f_{030}, x_{0} f_{030}
\end{array}\right\}, \\
& \widehat{F}_{J}^{(4)}=\left\{x_{2}^{2} f_{011}, x_{2} x_{1} f_{011}, x_{2} x_{0} f_{011}, x_{2} f_{030}\right\}, \\
& S F_{J}^{(4)}=\left\{\begin{array}{l}
x_{2}^{2} f_{011}-x_{2} x_{1} f_{002}, x_{2} x_{1} f_{011}-x_{1}^{2} f_{002}, \\
x_{2} x_{0} f_{011}-x_{1} x_{0} f_{002}, x_{2} f_{030}-x_{1}^{2} f_{011}
\end{array}\right\} .
\end{aligned}
$$


In order to study the sets of polynomials $\widetilde{F}_{J}^{(s)}$ and the module $\mathcal{N}(J, I)$, we need to know explicitly the $J$-marked set, so let us consider, for instance, $F_{J}=\left\{f_{002}, f_{011}, f_{030}\right\}$, where

$$
\begin{aligned}
& f_{002}=x_{2}^{2}+3 x_{1}^{2}-x_{2} x_{0}+x_{1} x_{0}, \\
& f_{011}=x_{2} x_{1}-x_{1} x_{0}, \\
& f_{030}=x_{1}^{3}-3 x_{1}^{2} x_{0},
\end{aligned}
$$

and let $I:=\left(F_{J}\right)$. For $s=2$, we have $\widetilde{F}_{J}^{(2)}=F_{J}^{(2)}$ and $\mathcal{N}(J, I)=\emptyset$.

$(s=3)$. In order to construct $\widetilde{F}_{J}^{(3)}$, we must determine the equivalence classes of monomials in the quotient $A\left[x_{0}, x_{1}, x_{2}\right]_{3} /\left\langle F_{J}^{(3)}\right\rangle \simeq$ $\left\langle\mathcal{N}(J)_{3}\right\rangle$. If $h \in A\left[x_{0}, x_{1}, x_{2}\right]_{s}$, we denote by $\bar{h}$ its class in the quotient

$$
A\left[x_{0}, x_{1}, x_{2}\right]_{s} /\left\langle F_{J}^{(s)}\right\rangle \text {. }
$$

Following the strategy of the proof of Theorem 1.7, we examine the monomials of $J_{3}$ in increasing order with respect to the Lex ordering.

$$
\begin{aligned}
& \overline{x_{1}^{3}} \stackrel{-f_{030}}{=} \overline{3 x_{1}^{2} x_{0}} \quad \Longrightarrow \quad \widetilde{f}_{030}=f_{030}, \\
& {\overline{x_{2} x_{1} x_{0}}}^{-x_{0} f_{011}} \overline{x_{1} x_{0}^{2}} \Longrightarrow \widetilde{f}_{111}=x_{0} f_{011} \text {, } \\
& \overline{x_{2} x_{1}^{2}} \stackrel{-x_{1} f_{011}}{=} \overline{x_{1}^{2} x_{0}} \Longrightarrow \widetilde{f}_{021}=x_{1} f_{011} \text {, } \\
& \overline{x_{2}^{2} x_{0}} \stackrel{-x_{0} f_{002}}{=} \overline{-3 x_{1}^{2} x_{0}+x_{2} x_{0}^{2}-x_{1} x_{0}^{2}} \Longrightarrow \tilde{f}_{102}=x_{0} f_{002} \text {, } \\
& \overline{x_{2}^{2} x_{1}}-x_{1} \stackrel{f_{002}}{-3 x_{1}^{3}+x_{2} x_{1} x_{0}-x_{1}^{2} x_{0}} \stackrel{\widetilde{f}_{030}}{=} \overline{x_{2} x_{1} x_{0}-10 x_{1}^{2} x_{0}} \\
& \stackrel{-\widetilde{f}_{11}}{=} \overline{-10 x_{1}^{2} x_{0}+x_{1} x_{0}^{2}} \Longrightarrow \widetilde{f}_{012}=x_{1} f_{002}-3 f_{030}+x_{0} f_{011}, \\
& \overline{x_{2}^{3}}-x_{\underline{2}}^{=} f_{002} \overline{-3 x_{2} x_{1}^{2}+x_{2}^{2} x_{0}-x_{2} x_{1} x_{0}} 3 \stackrel{\widetilde{f}_{021}}{=} \overline{x_{2}^{2} x_{0}-x_{2} x_{1} x_{0}-3 x_{1}^{2} x_{0}} \\
& -\stackrel{\widetilde{f}_{102}}{=} \overline{-x_{2} x_{1} x_{0}-6 x_{1}^{2} x_{0}+x_{2} x_{0}^{2}-x_{1} x_{0}^{2}} \\
& \stackrel{\widetilde{f}_{\underline{111}}}{=-6 x_{1}^{2} x_{0}+x_{2} x_{0}^{2}-2 x_{1} x_{0}^{2}} \\
& \Longrightarrow \tilde{f}_{003}=x_{2} f_{002}-3 x_{1} f_{011}+x_{0} f_{002}-x_{0} f_{011} .
\end{aligned}
$$

To determine $\mathcal{N}(J, I)_{3}$, we can compute the class of the polynomial of $S F_{J}^{(3)}$ in

$$
A\left[x_{0}, x_{1}, x_{2}\right]_{3} /\left\langle F_{J}^{(3)}\right\rangle=A\left[x_{0}, x_{1}, x_{2}\right]_{3} /\left\langle\widetilde{F}_{J}^{(3)}\right\rangle:
$$




$$
\overline{x_{2} f_{011}-x_{1} f_{002}}=\overline{-3 x_{1}^{3}-x_{1}^{2} x_{0}} \stackrel{3 \widetilde{f}_{030}}{=} \overline{-10 x_{1}^{2} x_{0}}
$$

so that $\mathcal{N}(J, I)_{3}=\left\langle 10 x_{1}^{2} x_{0}\right\rangle$.

$(s=4)$. Repeating the same procedure applied for $s=3$, we obtain:

$$
\begin{aligned}
\tilde{f}_{130} & =x_{0} f_{030}=x_{1}^{3} x_{0}-3 x_{1}^{2} x_{0}^{2}, \\
\tilde{f}_{040} & =x_{1} f_{030}-b x_{0} f_{030}=x_{1}^{4}-9 x_{1}^{2} x_{0}^{2}, \\
\widetilde{f}_{211} & =x_{0}^{2} f_{011}=x_{2} x_{1} x_{0}^{2}-x_{1} x_{0}^{3} \\
\widetilde{f}_{121} & =x_{1} x_{0} f_{011}=x_{2} x_{1}^{2} x_{0}-x_{1}^{2} x_{0}^{2}, \\
\widetilde{f}_{031} & =x_{1}^{2} f_{011}+x_{0} f_{030}=x_{2} x_{1}^{3}-3 x_{1}^{2} x_{0}^{2}, \\
\widetilde{f}_{202} & =x_{0}^{2} f_{002}=x_{2}^{2} x_{0}^{2}+3 x_{1}^{2} x_{0}^{2}-x_{2} x_{0}^{3}+x_{1} x_{0}^{3}, \\
\tilde{f}_{112} & =x_{1} x_{0} f_{002}-3 x_{0} f_{030}+x_{0}^{2} f_{211}=x_{2}^{2} x_{1} x_{0}+10 x_{1}^{2} x_{0}^{2}-x_{1} x_{0}^{3}, \\
\widetilde{f}_{022} & =x_{1}^{2} f_{002}-3 x_{1} f_{030}+x_{1} x_{0} f_{011}-10 x_{0} f_{030}=x_{2}^{2} x_{1}^{2}+29 x_{1}^{2} x_{0}^{2}, \\
\widetilde{f}_{103}= & x_{2} x_{0} f_{002}-3 x_{1} x_{0} f_{011}+x_{0}^{2} f_{002}-x_{0}^{2} f_{011} \\
= & x_{2}^{3} x_{0}+6 x_{1}^{2} x_{0}^{2}-x_{2} x_{0}^{3}+2 x_{1} x_{0}^{3}, \\
\widetilde{f}_{013}= & x_{2} x_{1} f_{002}-3 x_{1}^{2} f_{011}+x_{1} x_{0} f_{002}-x_{1} x_{0} f_{011}-6 x_{0} f_{030}+x_{0}^{2} f_{011} \\
= & x_{2}^{3} x_{1}+20 x_{1}^{2} x_{0}^{2}-x_{1} x_{0}^{3}, \\
\widetilde{f}_{004}= & x_{2}^{2} f_{002}-3 x_{1}^{2} f_{002}+9 x_{1} f_{030}+x_{2} x_{0} f_{002}-x_{1} x_{0} f_{002}-6 x_{1} x_{0} f_{011} \\
& +33 x_{0} f_{030}+x_{0}^{2} f_{002}-2 x_{0}^{2} f_{011}=x_{2}^{4}-91 x_{1}^{2} x_{0}^{2}-x_{2} x_{0}^{3}+3 x_{1} x_{0}^{3} .
\end{aligned}
$$

Moreover, $\mathcal{N}(J, I)_{4}=\left\langle 10 x_{1}^{2} x_{0}^{2}\right\rangle$ and, as in the quotient $A\left[x_{0}, x_{1}, x_{2}\right]_{4} /$ $\left\langle F_{J}^{(4)}\right\rangle$, we have

$$
\begin{array}{ll}
\overline{x_{2}^{2} f_{011}-x_{2} x_{1} f_{002}}=\overline{-10 x_{1}^{2} x_{0}^{2}}, & \overline{x_{2} x_{1} f_{011}-x_{1}^{2} f_{002}}=\overline{-30 x_{1}^{2} x_{0}^{2}}, \\
\overline{x_{2} x_{0} f_{011}-x_{1} x_{0} f_{002}}=\overline{-10 x_{1}^{2} x_{0}^{2}}, & \overline{x_{2} f_{030}-x_{1}^{2} f_{011}}=\overline{0} .
\end{array}
$$

Therefore, $F_{J}$ is not a $J$-marked basis unless $10=0$ in $A$ (cf., Theorem 1.7).

We conclude this section by giving a characterization of a $J$-marked basis $F_{J}$ that takes into account the homogeneous pieces $\left(F_{J}\right)_{s}$ of the ideal it generates for a limited number of degrees. The following statement is clearly inspired by Gotzmann's persistence theorem, but we emphasize that the proof is independent from that result. 
Theorem 1.10. Let $J$ be a strongly stable ideal, $m$ the maximum degree of monomials in its minimal monomial basis $\mathcal{B}_{J}$ and $I$ the ideal in $A[\mathbf{x}]$ generated by a $J$-marked set $F_{J}$. The following are equivalent:

(i) $F_{J}$ is a $J$-marked basis;

(ii) as an $A$-module, $I_{s}=\left\langle F_{J}^{(s)}\right\rangle$ for every $s \leq m+1$;

(iii) as an $A$-module, $I_{s}=\left\langle\widetilde{F}_{J}^{(s)}\right\rangle$ for every $s \leq m+1$;

(iv) $\mathcal{N}(J, I)_{s}=0$ for every $s \leq m+1$.

Proof.

(i) $\Rightarrow$ (ii). Straightforward by Theorem 1.7 (vi).

(ii) $\Rightarrow$ (i). We want to prove that, for every $s, A[\mathbf{x}]_{s}=I_{s} \oplus\left\langle\mathcal{N}(J)_{s}\right\rangle$. This is true for $s \leq m+1$ by hypothesis. By Theorem 1.7 (ii)-(iii), we know that

$$
A[\mathbf{x}]_{s}=\left\langle F_{J}^{(s)}\right\rangle \oplus\left\langle\mathcal{N}(J)_{s}\right\rangle
$$

and

$$
\left\langle F_{J}^{(s)}\right\rangle \subseteq I_{s}
$$

so we need to prove $I_{s} \subseteq\left\langle F_{J}^{(s)}\right\rangle$. Let us assume that this is not true, and let $t$ be the minimum degree for which $I_{t} \nsubseteq\left\langle F_{J}^{(t)}\right\rangle$. Note that

$$
t \geq m+2>m
$$

and

$$
I_{t}=x_{0} I_{t-1}+\cdots+x_{n} I_{t-1} .
$$

Since $I_{t-1}=\left\langle F_{J}^{(t-1)}\right\rangle$, there should exist a variable $x_{i}$ such that $x_{i} I_{t-1} \nsubseteq\left\langle F_{J}^{(t)}\right\rangle$, or equivalently, $x_{i} F_{J}^{(t-1)} \nsubseteq\left\langle F_{J}^{(t)}\right\rangle$. Assume that $x_{i}$ has the minimum index and take a polynomial $x^{\delta} f_{\alpha} \in F_{J}^{(t-1)}$, with $x^{\alpha}=\operatorname{Ht}\left(f_{\alpha}\right) \in \mathcal{B}_{J}$, such that $x_{i} x^{\delta} f_{\alpha} \notin\left\langle F_{J}^{(t)}\right\rangle$. The variable $x_{i}$ must be greater than min $x^{\alpha}$, since otherwise, $x_{i} x^{\delta} f_{\alpha} \in F_{J}^{(t)}$. Moreover, $|\delta|>0$ since $t-1>m$. Let

$$
x_{j}=\max x^{\delta} \leq \min x^{\alpha}<x_{i}
$$

and

$$
x^{\delta^{\prime}}=\frac{x^{\delta}}{x_{j}} .
$$


The polynomial $x_{i} x^{\delta^{\prime}} f_{\alpha}$ is contained in $I_{t-1}$, while $x_{j}\left(x_{i} x^{\delta^{\prime}} f_{\alpha}\right)=$ $x_{i} x^{\delta} f_{\alpha}$ is not contained in $\left\langle F_{J}^{(t-1)}\right\rangle$, contradicting the minimality of $i$.

(ii) $\Leftrightarrow$ (iii) $\Leftrightarrow$ (iv). Straightforward by Theorem 1.7.

2. Definition and representability of marked functors. We follow the notation for functors used in [16]. The main object of interest in the present paper is the set,

$$
\underline{\mathbf{M f}}_{J}(A):=\left\{\text { ideals } I \subseteq A[\mathbf{x}] \mid A[\mathbf{x}]=I \oplus{ }_{A}\langle\mathcal{N}(J)\rangle\right\},
$$

which is defined for every Noetherian ring $A$ and every strongly stable ideal $J \subseteq A[\mathbf{x}]$. In this section, we will prove that this construction is in fact functorial, i.e., $\underline{\mathbf{M f}}_{J}(A)$ is the evaluation in the Noetherian ring $A$ of a functor

$$
\underline{\text { Mf }}_{J}: \text { Noeth-Rings } \longrightarrow \underline{\text { Sets. }}
$$

Now we will describe the elements of any $\underline{\mathbf{M f}}_{J}(A)$ in terms of the notion of a $J$-marked basis, discussed in the previous section. This will be a key point in proving its functoriality.

Proposition 2.1. Let $J$ be a strongly stable ideal, and let $I$ be an element of $\underline{\mathbf{M f}}_{J}(A)$.

(i) The ideal I contains a unique $J$-marked set $F_{J}$.

(ii) $I=\left(F_{J}\right)$ and $F_{J}$ is the unique $J$-marked basis contained in $I$.

Proof.

(i) Let $x^{\alpha}$ be a minimal generator of $J$, and consider its image by the projection $A[\mathbf{x}] \stackrel{\pi_{I}}{\longrightarrow} A[\mathbf{x}] / I$. Since $A[\mathbf{x}]_{|\alpha|} / I_{|\alpha|} \simeq\left\langle\mathcal{N}(J)_{|\alpha|}\right\rangle, \pi_{I}\left(x^{\alpha}\right)$ is given by a linear combination $\sum c_{\alpha \beta} x^{\beta}$ of the monomials, $x^{\beta} \in$ $\mathcal{N}(J)_{|\alpha|}$. Therefore, ker $\pi$ contains a unique homogeneous polynomial $f_{\alpha}=x^{\alpha}-\sum c_{\alpha \beta} x^{\beta}$ with head term $x^{\alpha}$. The collection of all $f_{\alpha}$, for $x^{\alpha} \in \mathcal{B}_{J}$, is the unique $J$-marked set.

(ii) Starting from $F_{J}$, we can construct, for every degree $s$, the sets of polynomials $F_{J}^{(s)}$ and $\widetilde{F}_{J}^{(s)}$ as in Theorem 1.10. Recall that they are both contained in the ideal $\left(F_{J}\right) \subseteq I$. In order to show that $F_{J}$ is a $J$-marked basis and generates $I$, we observe that, for every $s,\left\langle F_{J}^{(s)}\right\rangle \subseteq I_{s}$ and $\left\langle F_{J}^{(s)}\right\rangle \oplus\left\langle\mathcal{N}(J)_{s}\right\rangle=A[\mathbf{x}]_{s}$ by Theorem 1.7 (ii). 
Moreover, $I_{s} \oplus\left\langle\mathcal{N}(J)_{s}\right\rangle=A[\mathbf{x}]_{s}$, since $I \in \underline{\mathbf{M f}}_{J}(A)$. Therefore, $I_{s}=\left\langle F_{J}^{(s)}\right\rangle=\left(F_{J}^{(s)}\right)_{s}$ in every degree $s$. Finally, $F_{J}$ is a $J$-marked basis by Theorem 1.7 (v)-(vi) and is unique by (i).

Remark 2.2. We emphasize that uniqueness is not true for a $J$ marked set generating an ideal $I \notin \underline{\mathbf{M f}}_{J}(A)$. For instance, consider the strongly stable ideal $J=\left(x_{2}^{2}, x_{2} x_{1}, x_{1}^{3}\right) \subseteq \mathbb{Z}\left[x_{0}, x_{1}, x_{2}\right]$. The $J$ marked set $F_{J}=\left\{x_{2}^{2}+x_{0}^{2}, x_{2} x_{1}, x_{1}^{3}\right\}$ defines an ideal $I=\left(F_{J}\right)$ not contained in $\underline{\mathbf{M f}}_{J}(\mathbb{Z})$ as $x_{1} x_{0}^{2}=x_{1}\left(x_{2}^{2}+x_{0}^{2}\right)-x_{2}\left(x_{2} x_{1}\right) \in I \cap \mathcal{N}(J)$. In fact, the ideal $I$ is generated by infinitely many $J$-marked sets $\left\{x_{2}^{2}+x_{0}^{2}, x_{2} x_{1}, x_{1}^{3}+a x_{1} x_{0}^{2}\right\}, a \in \mathbb{Z}$.

As a consequence of the previous result, we are now able to give a new description of $\underline{\mathbf{M f}}{ }_{J}(A)$ :

$\underline{\operatorname{Mf}}_{J}(A)=\{$ ideals $I \subseteq A[\mathbf{x}] \mid I$ is generated by a $J$-marked basis $\}$.

For every strongly stable ideal $J$, let us consider the map between the category of Noetherian rings to the category of sets

$$
\underline{\text { Mf }}_{J}: \text { Noeth-Rings } \longrightarrow \underline{\text { Sets }}
$$

that associates the set $\underline{\mathbf{M f}}{ }_{J}(A)$ to a Noetherian ring $A$ and the map

$$
\begin{aligned}
\underline{\mathbf{M f}}_{J}(\phi): \underline{\mathbf{M f}}_{J}(A) & \longrightarrow \underline{\mathbf{M f}}_{J}(B) \\
I & \longmapsto I \otimes_{A} B
\end{aligned}
$$

to a morphism $\phi: A \rightarrow B$.

Proposition 2.3. For every strongly stable ideal $J, \underline{\mathbf{M f}}_{J}$ is a functor.

Proof. Consider the $J$-marked basis $F_{J, A}$ generating the ideal $I \in$ $\underline{\mathbf{M f}}_{J}(A)$. Any morphism $\phi: A \rightarrow B$ gives the structure of an $A$-module to $B$. Thus, tensoring $I$ by $B$ leads to the following transformation on the $J$-marked basis $F_{J, A}$ :

$$
f_{\alpha, A}=x^{\alpha}-\sum c_{\alpha \beta} x^{\beta} \in F_{J, A} \longmapsto f_{\alpha, B}=x^{\alpha}-\sum \phi\left(c_{\alpha \beta}\right) x^{\beta} .
$$

Since $\phi\left(1_{A}\right)=1_{B}$, the set $F_{J, B}:=\left\{f_{\alpha, B} \mid f_{\alpha, A} \in F_{J, A}\right\}$ is still a $J$ marked set. Finally, $F_{J, B}$ is a $J$-marked basis since the tensor product by $\otimes_{A} B$ of a direct sum of free $A$-modules is a direct sum of free $B$ modules. 
Now we discuss a necessary condition for this functor to be representable.

Lemma 2.4. For every strongly stable ideal $J, \underline{\mathbf{M f}}_{J}$ is a Zariski sheaf.

Proof. Let $A$ be a Noetherian ring and $U_{i}=\operatorname{Spec} A_{a_{i}}, i=$ $1, \ldots, s$, an open cover of $\operatorname{Spec} A$, which is equivalent to requiring that $\left(a_{1}, \ldots, a_{s}\right)=A$. Consider a set of ideals $I_{i} \in \underline{\mathbf{M f}}_{J}\left(A_{a_{i}}\right)$ such that, for any pair of indices $i \neq j$,

$$
I_{i j}:=I_{i} \otimes_{A_{a_{i}}} A_{a_{i} a_{j}}=I_{j} \otimes_{A_{a_{j}}} A_{a_{i} a_{j}} \in \underline{\mathbf{M f}}_{J}\left(A_{a_{i} a_{j}}\right) .
$$

We need to show that there exists a unique ideal $I \in \underline{\mathbf{M f}}_{J}(A)$ such that $I_{i}=I \otimes_{A} A_{a_{i}}$ for every $i$.

Let us consider the $J$-marked bases associated to $I_{i}$ :

$$
F_{J, i}=\left\{f_{\alpha, i}=x^{\alpha}-\sum_{x^{\beta} \in \mathcal{N}(J)_{|\alpha|}} c_{\alpha \beta}^{(i)} x^{\beta} \in A_{a_{i}}[\mathbf{x}] \mid x^{\alpha} \in \mathcal{B}_{J}\right\},
$$

where $I_{i}=\left(F_{J, i}\right) \subseteq A_{a_{i}}[\mathbf{x}]$, for all $i=1, \ldots, s$. By assumption, for each $x^{\alpha} \in \mathcal{B}_{J}$ and for each pair of indices $i \neq j$, the polynomials $f_{\alpha, i}$ and $f_{\alpha, j}$ coincide on $A_{a_{i} a_{j}}[\mathbf{x}]$. By the sheaf axiom for the quasi-coherent sheaf $\widetilde{A[\mathbf{x}]}$ on $\operatorname{Spec} A$, we know that there exists a unique polynomial $f_{\alpha} \in A[\mathbf{x}]$ whose image in $A_{a_{i}}[\mathbf{x}]$ is $f_{\alpha, i}$ for every $i$. The polynomial $f_{\alpha}$ turns out to be monic. In fact, if $c$ is the coefficient of $x^{\alpha}$, then its image in $A_{a_{i}}$ is $1_{A_{a_{i}}}$, so that $\left(c-1_{A}\right) a_{i}^{k}=0$ for some integer $k$. Thus, $c=1_{A}$ since $\left(a_{1}^{k}, \ldots, a_{s}^{k}\right)=A$. The collection of polynomials $\left\{f_{\alpha}: x^{\alpha} \in \mathcal{B}_{J}\right\}$ forms a $J$-marked basis.

Now we prove that the functor $\underline{\mathbf{M f}}_{J}$ is representable, explicitly finding the affine scheme $\mathbf{M f}_{J}$ representing it. To do that, we apply the previous theorems that describe which conditions on the coefficients of polynomials in a $J$-marked set guarantee that the marked set is a $J$-marked basis.

We obtain $\mathbf{M f}_{J}$ as a closed subscheme of an affine scheme of a suitable dimension depending on $J$.

Notation 2.5. Let $J$ be any strongly stable monomial ideal in $\mathbb{Z}[\mathbf{x}]$. Then: 
- $\mathbf{C}$ is the set of variables of the coordinate ring of the affine scheme $\mathbb{A}^{N}=\operatorname{Spec} \mathbb{Z}[\mathbf{C}]$, where

$$
N=\sum_{x^{\alpha} \in \mathcal{B}_{J}}\left|\mathcal{N}(J)_{|\alpha|}\right| .
$$

We consider the variables in $\mathbf{C}$ indexed as $C_{\alpha \beta}$ where the multiindex $\alpha$ corresponds to $x^{\alpha} \in \mathcal{B}_{J}$ and the multi-index $\beta$ to $x^{\beta} \in \mathcal{N}(J)_{|\alpha|}$.

- $\mathcal{I}$ is the ideal in $\mathbb{Z}[\mathbf{C}][\mathbf{x}]=\mathbb{Z}[\mathbf{C}] \otimes_{\mathbb{Z}} \mathbb{Z}[\mathbf{x}]$ generated by the following $J$-marked set:

$$
\mathcal{F}_{J}:=\left\{x^{\alpha}-\sum_{x^{\beta} \in \mathcal{N}(J)_{|\alpha|}} C_{\alpha \beta} x^{\beta} \in \mathbb{Z}[\mathbf{C}][\mathbf{x}] \mid x^{\alpha} \in \mathcal{B}_{J}\right\} .
$$

- $\mathfrak{I}_{J}$ is the ideal in $\mathbb{Z}[\mathbf{C}]$ generated by the $\mathbf{x}$-coefficients of the polynomials in $\mathcal{N}(J, \mathcal{I})$.

- Every $J$-marked set

$$
F_{J}=\left\{f_{\alpha}=x^{\alpha}-\sum_{x^{\beta} \in \mathcal{N}(J)_{|\alpha|}} c_{\alpha \beta} x^{\beta} \mid x^{\alpha} \in \mathcal{B}_{J}\right\}
$$

in $A[\mathbf{x}]$ is uniquely identified by the coefficients $c_{\alpha \beta} \in A$, or equivalently, by the ring homomorphism,

$$
\phi_{F_{J}}: \mathbb{Z}[\mathbf{C}] \longrightarrow A: C_{\alpha \beta} \longmapsto c_{\alpha \beta} .
$$

- Moreover, let $\phi_{F_{J}}[\mathbf{x}]: \mathbb{Z}[\mathbf{C}][\mathbf{x}] \rightarrow A[\mathbf{x}]$ be the canonical extension of $\phi_{F_{J}}$.

Theorem 2.6. In the notation above, the functor $\underline{\mathbf{M f}}_{J}$ is represented by $\mathbf{M f}_{J}:=\operatorname{Spec} \mathbb{Z}[\mathbf{C}] / \Im_{J}$. Therefore, a $J$-marked set $F_{J}$ is a $J$-marked basis if, and only if, $\phi_{F_{J}}$ factors.

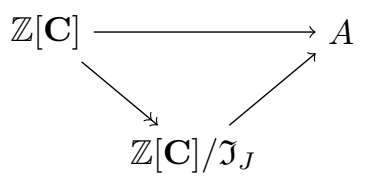


Proof. Let $A$ be any Noetherian ring, $F_{J}$ a $J$-marked set in $A[\mathbf{x}]$ and $I=\left(F_{J}\right) \subseteq A[\mathbf{x}]$. We obtain the statement proving that $F_{J}$ is a $J$-marked basis if, and only if, $\operatorname{ker} \phi_{F_{J}} \supseteq \mathfrak{I}_{J}$.

By definition, $\phi_{F_{J}}[\mathbf{x}]$ is the identity on monomials and $\phi_{F_{J}}[\mathbf{x}](\mathcal{I}) \subseteq$ $I$, so that $\phi_{F_{J}}[\mathbf{x}](\mathcal{N}(J, \mathcal{I})) \subseteq \mathcal{N}(J, I)$. If $F_{J}$ is a $J$-marked basis, then $\mathcal{N}(J, I)=0$; hence, $\operatorname{ker}\left(\phi_{F_{J}}\right) \supseteq \mathfrak{I}_{J}$.

On the other hand, if $\operatorname{ker}\left(\phi_{F_{J}}\right) \supseteq \mathfrak{I}_{J}$, then for every $s$, we have

$$
\begin{aligned}
\widehat{F}_{J}^{(s)} & =\phi_{F_{J}}[\mathbf{x}]\left(\widehat{\mathcal{F}}_{J}^{(s)}\right) \subseteq \phi_{F_{J}}[\mathbf{x}]\left(\mathcal{I}_{s}\right) \\
& =\phi_{F_{J}}[\mathbf{x}]\left(\left\langle\mathcal{F}_{J}^{(s)}\right\rangle \oplus \mathcal{N}(J, \mathcal{I})_{s}\right) \\
& =\phi_{F_{J}}[\mathbf{x}]\left(\left\langle\mathcal{F}_{J}^{(s)}\right\rangle\right) \subseteq{ }_{A}\left\langle F^{(s)}\right\rangle,
\end{aligned}
$$

so that

$$
I_{s}={ }_{A}\left\langle F_{J}^{(s)} \cup \widehat{F}_{J}^{(s)}\right\rangle \subseteq{ }_{A}\left\langle F_{J}^{(s)}\right\rangle \subseteq I_{s},
$$

and we conclude by applying Theorem 1.7 (v)-(vi).

In order to explicitly compute a finite set of generators of the ideal $\mathfrak{I}_{J}$, we can apply some of the previous results. By Theorem 1.10, we get the following simplification.

Corollary 2.7. For every strongly stable ideal $J$, the ideal $\mathfrak{I}_{J}$ is generated by the $\mathbf{x}$-coefficients of the polynomials in $\mathcal{N}(J, \mathcal{I})_{s}$ for every $s \leq m+1$, where $m$ is the maximum degree of monomials in $\mathcal{B}_{J}$.

Proof. Let $\mathfrak{I}^{\prime}$ be the ideal in $\mathbb{Z}[\mathbf{C}]$ generated by $\mathbf{x}$-coefficients of the polynomials in $\mathcal{N}(J, \mathcal{I})_{s}$ for every $s \leq m+1$. Obviously, $\mathfrak{I}^{\prime} \subseteq \mathfrak{I}_{J}$.

On the other hand, by Theorem 1.10 , the image of $\mathcal{F}_{J}$ in $\mathbb{Z}[\mathbf{C}] / \mathfrak{I}^{\prime} \otimes_{\mathbb{Z}}$ $\mathbb{Z}[\mathbf{x}]$ is a $J$-marked basis. Therefore, the map

$$
\mathbb{Z}[\mathbf{C}] \longrightarrow \mathbb{Z}[\mathbf{C}] / \mathfrak{I}^{\prime}
$$

factors through $\mathbb{Z}[\mathbf{C}] / \mathfrak{I}_{J}$.

We can obtain a set of generators of $\mathfrak{I}_{J}$ by computing a set of generators of the $\mathbb{Z}$-module $\mathcal{N}\left(J, \mathcal{F}_{J}\right)_{s}$ for each $s \leq m+1$ through a Gaussian reduction. This is the method applied, for instance, in [9].

A more efficient method is the one developed in [4], which is similar to the Buchberger algorithm for Gröbner bases. We will now describe 
this method and then prove that it, in fact, gives a set of generators of $\mathfrak{I}_{J}$, as claimed in [4].

Definition 2.8. Let $J$ be a strongly stable ideal, and let $F_{J}$ be a marked set. We say that a polynomial $g$ is a $J$-remainder if $\operatorname{Supp}(g) \cap J=\emptyset$. Given two polynomials $h$ and $g$, we say that $g$ can be obtained from $h$ by a step of $F_{J}$-reduction if $g=h-c f$ where $c$ is the coefficient in $h$ of a monomial $x^{\eta} \in J_{s}$ and $f$ is the unique polynomial in $F_{J}^{(s)}$ with $\operatorname{Ht}(f)=x^{\eta}$. We write

$$
h \stackrel{F_{J}^{(\cdot)}}{\longrightarrow} g,
$$

if $g$ arises from $h$ by a finite sequence of reductions as described above. Moreover, we write

$$
h \stackrel{F_{J}^{(\cdot)}}{\longrightarrow} g
$$

if $g$ is a $J$-remainder.

As proved in $[4,9]$, the procedure $\stackrel{F_{J}^{(\cdot)}}{\longrightarrow}$ is Noetherian, i.e., every sequence of $F_{J}$-reductions starting on a polynomial $h$ stops after a finite number of steps giving a $J$-remainder polynomial $g$. Indeed, each step of reduction $h \mapsto h-c f$ replaces a monomial $x^{\eta}=x^{\alpha} *_{J} x^{\gamma}$ in support of $h$ with $x^{\gamma} T\left(f_{\alpha}\right), f_{\alpha} \in F_{J}$. Since $T\left(f_{\alpha}\right) \in\langle\mathcal{N}(J)\rangle$, every monomial appearing in $x^{\gamma} T\left(f_{\alpha}\right)$ either is in $\mathcal{N}(J)$ or has the decomposition $x^{\gamma} x^{\beta}=x^{\alpha^{\prime}} *_{J} x^{\delta}$ with $x^{\delta}<_{\text {Lex }} x^{\gamma}$. This leads to the conclusion since $<_{\text {Lex }}$ is a well ordering on monomials.

We can find many different sequences of steps of reduction starting from a given polynomial $h$, but the $J$-remainder polynomial $g$ is unique. In fact, if $h$ is a homogeneous polynomial of degree $s$,

$$
h \stackrel{F_{J}^{(\cdot)}}{\longrightarrow} g \text { and } h \stackrel{F_{J}^{(\cdot)}}{\longrightarrow} g^{\prime},
$$

then

$$
g-g^{\prime}=(g-h)-\left(g^{\prime}-h\right) \in\left\langle F_{J}^{(s)}\right\rangle,
$$

since $g-h, g^{\prime}-h \in\left\langle F_{J}^{(s)}\right\rangle$. By definition of the $J$-remainder, 


$$
g-g^{\prime} \in\left\langle\mathcal{N}(J)_{s}\right\rangle \text { and }\left\langle\mathcal{N}(J)_{s}\right\rangle \cap\left\langle F_{J}^{(s)}\right\rangle=\{0\}
$$

by Theorem 1.7 (ii).

Remark 2.9. In general, the marking cannot be performed with respect to a term ordering (see [9, Example 3.18]), so that the Noetherianity of the procedure is surprising. Indeed, it is well known that a general reduction process by a set of marked polynomials is Noetherian if, and only if, the marking is performed with respect to a term ordering (see [26]). The ultimate reason for this is our restriction that each monomial $x^{\eta} \in J$ is reduced by the unique polynomial $x^{\gamma} f_{\alpha} \in F_{J}^{(s)}$ such that $x^{\eta}=x^{\alpha} *_{J} x^{\gamma}$, as opposed to any polynomial $x^{\delta} f_{\beta} \in\left(F_{J}\right)$ such that $x^{\delta} x^{\beta}=x^{\eta}$.

Now we can give a characterization of a $J$-marked basis in terms of this reduction procedure and $S$-polynomials.

Definition 2.10. For marked polynomials $f_{\alpha}, f_{\beta}$ in a $J$-marked set, we call $S\left(f_{\alpha}, f_{\beta}\right):=x^{\eta} f_{\alpha}-x^{\nu} f_{\beta}$ the $S$-polynomial of $f_{\alpha}$ and $f_{\beta}$, where $\left(x^{\eta},-x^{\nu}\right)$ is the minimal syzygy between $x^{\alpha}$ and $x^{\beta}$. We call EKpolynomial, and denote by $S^{\mathrm{EK}}\left(f_{\alpha}, f_{\beta}\right)$, an $S$-polynomial whose syzygy $\left(x^{\eta},-x^{\nu}\right)$ is of Eliahou-Kervaire type, i.e., $x^{\eta}$ is a single variable $x_{j}$ greater than $\min \left(x^{\alpha}\right)$ and $x_{j} x^{\alpha}=x^{\beta} *_{J} x^{\nu}$, see [11].

Note that, for every EK-polynomial $x_{j} f_{\alpha}-x^{\nu} f_{\beta} \in A[\mathbf{x}]_{s}$, we have $x_{j} f_{\alpha} \in \widehat{F}_{J}^{(s)}$ and $x^{\nu} f_{\beta} \in F_{J}^{(s)}$.

Theorem 2.11. Let $J$ be a strongly stable ideal, and let $F_{J}$ be a Jmarked set. The following are equivalent:

(i) $F_{J}$ is a J-marked basis;

(ii) $S^{\mathrm{EK}}\left(f_{\alpha}, f_{\beta}\right) \stackrel{F_{J}^{(\cdot)}}{\longrightarrow}$ 0 , for all $S^{\mathrm{EK}}\left(f_{\alpha}, f_{\beta}\right)$ with $f_{\alpha}, f_{\beta} \in F_{J}$;

(iii) $x_{i} f_{\alpha} \stackrel{F_{J}^{(\cdot)}}{\longrightarrow}$ 0, for all $f_{\alpha} \in F_{J}$ and $x_{i}>\min x^{\alpha}$. 


\section{Proof.}

(i) $\Leftrightarrow$ (ii). The EK-syzygies are a basis of the syzygies of $J$, so (ii) ensures that every other syzygy between the two monomials $x^{\alpha}, x^{\beta} \in$ $\mathcal{B}_{J}$ lifted to the corresponding marked polynomials $f_{\alpha}$ and $f_{\beta}$ has $J$ remainder equal to 0 . Since these syzygies are exactly the generators of the module $S F_{J}^{(s)}$ for all $s$, (ii) is equivalent to $\left(F_{J}\right)_{s}=\left\langle F_{J}^{(s)}\right\rangle$ for every $s$, hence to (i) by Theorem 1.7.

(iii) $\Leftrightarrow$ (ii). The two reductions agree.

Remark 2.12. Notice that it is possible to prove the equivalence between Theorem 2.11 (i) and (iii) directly from the properties of the reduction $\stackrel{F_{J}^{(\cdot)}}{\longrightarrow}$, as was done in $[4]$.

We now show how a set of generators of $\mathfrak{I}_{J}$ can be computed using Theorem 2.11. We consider the $J$-marked set $\mathcal{F}_{J}$ given in (2.4) and use the marked sets $\widetilde{\mathcal{F}}_{J}^{(s)}$ in order to perform the polynomial reduction in each degree $s$. The elements of $\widetilde{\mathcal{F}}_{J}^{(s)}$ take the shape

$$
\tilde{f}_{\gamma}=x^{\gamma}-\sum_{x^{\delta} \in \mathcal{N}(J)_{s}} D_{\gamma \delta} x^{\delta}, \quad \text { for all } x^{\gamma} \in J_{s},
$$

with coefficients $D_{\gamma \delta} \in \mathbb{Z}[\mathbf{C}]$.

Let $S^{\mathrm{EK}}\left(f_{\alpha}, f_{\alpha^{\prime}}\right)$ be an EK-polynomial with $f_{\alpha}, f_{\alpha^{\prime}} \in \mathcal{F}_{J}$. Assume that $\operatorname{deg} S^{\mathrm{EK}}\left(f_{\alpha}, f_{\alpha^{\prime}}\right)=s$. We can decompose it as

$$
S^{\mathrm{EK}}\left(f_{\alpha}, f_{\alpha^{\prime}}\right)=x^{\eta} f_{\alpha}-x^{\eta^{\prime}} f_{\alpha^{\prime}}=\sum_{x^{\gamma} \in J_{s}} E_{\alpha \alpha^{\prime} \gamma} x^{\gamma}+\sum_{x^{\delta} \in \mathcal{N}(J)_{s}} E_{\alpha \alpha^{\prime} \delta} x^{\delta},
$$

where the coefficients in the first summand are equal to:

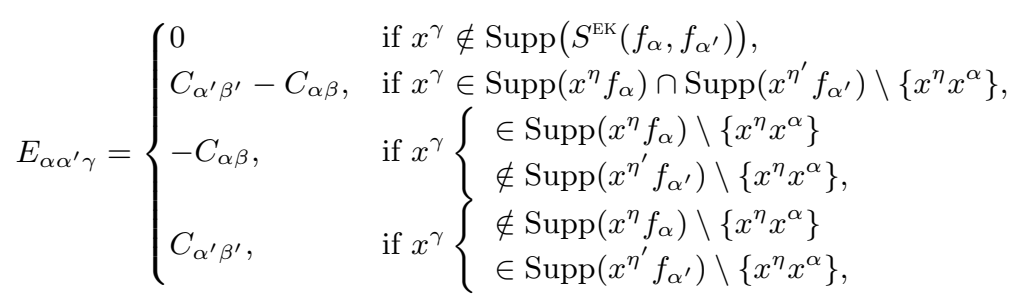


and the same possibilities hold for the coefficients $E_{\alpha \alpha^{\prime} \delta}$ in the second summand considering $x^{\delta}$ instead of $x^{\gamma}$ in the previous formula.

The $\mathcal{F}_{J^{-}}$-reduction of $S^{\mathrm{EK}}\left(f_{\alpha}, f_{\alpha^{\prime}}\right)$ is

$$
S^{\mathrm{EK}}\left(f_{\alpha}, f_{\alpha^{\prime}}\right) \stackrel{\mathcal{F}_{J}^{(\cdot)}}{\longrightarrow} \sum_{x^{\delta} \in \mathcal{N}(J)_{s}}\left(E_{\alpha \alpha^{\prime} \delta}+\sum_{x \gamma \in J_{s}} E_{\alpha \alpha^{\prime} \gamma} D_{\gamma \delta}\right) x^{\delta} .
$$

For any $\alpha, \alpha^{\prime}$ such that $x^{\alpha}, x^{\alpha^{\prime}} \in \mathcal{B}_{J}$ are involved in a syzygy of Eliahou-Kervaire type, we set

$$
\begin{gathered}
P_{\alpha \alpha^{\prime}}^{\delta}:=E_{\alpha \alpha^{\prime} \delta}+\sum_{x^{\gamma} \in J_{s}} E_{\alpha \alpha^{\prime} \gamma} D_{\gamma \delta}, \\
s=\operatorname{deg} S^{\mathrm{EK}}\left(f_{\alpha}, f_{\alpha^{\prime}}\right), \quad \text { for all } x^{\delta} \in \mathcal{N}(J)_{s} .
\end{gathered}
$$

Corollary 2.13. Let $J$ be a strongly stable ideal, and let $\mathfrak{I}_{J}$ be the ideal in $\mathbb{Z}[\mathbf{C}]$ given in Theorem 2.11. Then $\mathfrak{I}_{J}$ is the ideal generated by all polynomials $P_{\alpha \alpha^{\prime}}^{\delta}$ described in (2.5).

Proof. Let $\mathfrak{I}^{\prime}$ be the ideal generated by such polynomials $P_{\alpha \alpha^{\prime}}^{\delta}$. The inclusion $\mathfrak{I}^{\prime} \subseteq \mathfrak{I}_{J}$ follows directly from the construction, since the above polynomials are $\mathbf{x}$-coefficients of elements in $\mathcal{N}\left(J,\left(\mathcal{F}_{J}\right)\right)$.

For the opposite inclusion, we again consider the $J$-marked set $\overline{\mathcal{F}}_{J}$ image of $\mathcal{F}_{J}$ in $\mathbb{Z}[\mathbf{C}] / \mathfrak{I}^{\prime}$. By construction, $\overline{\mathcal{F}}_{J}$ satisfy condition (iii) of Theorem 2.11 , so that it is a $J$-marked basis. Therefore, $\mathbb{Z}[\mathbf{C}] \rightarrow$ $\mathbb{Z}[\mathbf{C}] / \mathfrak{I}^{\prime}$ factors as

$$
\mathbb{Z}[\mathbf{C}] \longrightarrow \mathbb{Z}[\mathbf{C}] / \mathfrak{I}_{J} \longrightarrow \mathbb{Z}[\mathbf{C}] / \mathfrak{I}^{\prime}
$$

and

$$
\mathfrak{I}_{J} \subseteq \mathfrak{I}^{\prime}
$$

To determine equations defining $\mathbf{M f}_{J}$ we can use Corollary 2.13, namely, the criterion for marked bases in terms of syzygies given in Theorem 2.11 that was first introduced in [9] and refined in [4] in terms of EK-syzygies. In particular, Theorem 2.11 and Corollary 2.13 give new proofs in terms of marked functors of [4, Corollary 4.6] and [9, Theorem 4.1]. 
Example 2.14. Let us compute the equations defining the scheme representing the functor $\underline{\mathbf{M f}}_{J}$ with $J=\left(x_{2}^{2}, x_{2} x_{1}, x_{1}^{3}\right) \subseteq \mathbb{Z}\left[x_{0}, x_{1}, x_{2}\right]$. We start by considering the marked set

$$
\begin{aligned}
& f_{002}=x_{2}^{2}+C_{002,020} x_{1}^{2}+C_{002,101} x_{2} x_{0}+C_{002,110} x_{1} x_{0}+C_{002,200} x_{0}^{2} \\
& f_{011}=x_{2} x_{1}+C_{011,020} x_{1}^{2}+C_{011,101} x_{2} x_{0}+C_{011,110} x_{1} x_{0}+C_{011,200} x_{0}^{2} \\
& f_{030}=x_{1}^{3}+C_{030,120} x_{1}^{2} x_{0}+C_{030,201} x_{2} x_{0}^{2}+C_{030,210} x_{1} x_{0}^{2}+C_{030,300} x_{0}^{3} .
\end{aligned}
$$

There are two EK-polynomials:

$$
\begin{aligned}
S^{\mathrm{EK}}\left(f_{011}, f_{002}\right)= & x_{2} f_{011}-x_{1} f_{002}=C_{011,020} x_{2} x_{1}^{2}-C_{002,020} x_{1}^{3} \\
& +C_{011,101} x_{2}^{2} x_{0}+\left(-C_{002,101}+C_{011,110}\right) x_{2} x_{1} x_{0} \\
& -C_{002,110} x_{1}^{2} x_{0}+C_{011,200} x_{2} x_{0}^{2}-C_{002,200} x_{1} x_{0}^{2}, \\
S^{\mathrm{EK}}\left(f_{030}, f_{011}\right)= & x_{2} f_{030}-x_{1}^{2} f_{011}=-C_{011,020} x_{1}^{4}+\left(-C_{011,101}+C_{030,120}\right) x_{2} x_{1}^{2} x_{0} \\
& -C_{011,110} x_{1}^{3} x_{0}+C_{030,201} x_{2}^{2} x_{0}^{2} \\
& +C_{030,210} x_{2} x_{1} x_{0}^{2}-C_{011,200} x_{1}^{2} x_{0}^{2}+C_{030,300} x_{2} x_{0}^{3} .
\end{aligned}
$$

\section{Since}

$$
\operatorname{Supp}\left(S^{\mathrm{EK}}\left(f_{011}, f_{002}\right)\right) \cap J=\left\{x_{2} x_{1}^{2}, x_{1}^{3}, x_{2}^{2} x_{0}, x_{2} x_{1} x_{0}\right\}
$$

and

$$
\operatorname{Supp}\left(S^{\mathrm{EK}}\left(f_{030}, f_{011}\right)\right) \cap J=\left\{x_{1}^{4}, x_{2} x_{1}^{2} x_{0}, x_{1}^{3} x_{0}, x_{2}^{2} x_{0}^{2}, x_{2} x_{1} x_{0}^{2}\right\},
$$

to perform the $\stackrel{\mathcal{F}_{J}^{(\cdot)}}{\longrightarrow}$ * reduction, we need some elements of $\widetilde{\mathcal{F}}_{J}^{(3)}$ and $\widetilde{\mathcal{F}}_{J}^{(4)}$. Reducing $S^{\mathrm{EK}}\left(f_{011}, f_{002}\right)$ by

$$
\begin{aligned}
\widetilde{f}_{111}= & x_{0} f_{011}, \quad \widetilde{f}_{102}=x_{0} f_{002}, \quad \widetilde{f}_{030}=f_{030}, \\
\widetilde{f}_{021}= & x_{1} f_{011}-C_{011,020} \widetilde{f}_{030}-C_{011,101} \\
\widetilde{f}_{111}= & x_{2} x_{1}^{2}+\left(-C_{011,020} C_{011,101}-C_{011,020} C_{030,120}+C_{011,110}\right) x_{1}^{2} x_{0} \\
& +\left(-C_{011,101}^{2}-C_{011,020} C_{030,201}\right) x_{2} x_{0}^{2} \\
& +\left(-C_{011,101} C_{011,110}-C_{011,020} C_{030,210}+C_{011,200}\right) x_{1} x_{0}^{2} \\
& +\left(-C_{011,101} C_{011,200}-C_{011,020} C_{030,300}\right) x_{0}^{3},
\end{aligned}
$$


we obtain

$$
\begin{gathered}
\underbrace{\left(\begin{array}{c}
C_{011,020}^{2} C_{011,101}+C_{011,020}^{2} C_{030,120}+C_{002,101} C_{011,020} \\
-C_{002,020} C_{011,101}-2 C_{011,020} C_{011,110} \\
+C_{002,020} C_{030,120}-C_{002,110}
\end{array}\right)} x_{1}^{2} x_{0} \\
+\underbrace{\left(\begin{array}{c}
C_{011,020} C_{011,101}^{2}+C_{011,020}^{2} C_{030,201}-C_{011,101} C_{011,110} \\
+C_{002,020} C_{030,201}+C_{011,200}
\end{array}\right)}_{P_{011,002}^{120}} x_{2}^{P_{011,002}^{201}} x_{0}^{2} \\
+\underbrace{\left(\begin{array}{c}
C_{011,020} C_{011,101} C_{011,110}+C_{011,020}^{2} C_{030,210}-C_{002,110} C_{011,101} \\
+C_{002,101} C_{011,110}-C_{011,110}^{2}-C_{011,020} C_{011,200} \\
+C_{002,020} C_{030,210}-C_{002,200}
\end{array}\right)}_{P_{011,002}^{210}} x_{1} x_{0}^{2} \\
+\underbrace{\left(\begin{array}{c}
C_{011,020} C_{011,101} C_{011,200}+C_{011,020}^{2} C_{030,300}-C_{002,200} C_{011,101} \\
+C_{002,101} C_{011,200}-C_{011,110} C_{011,200}+C_{002,020} C_{030,300}
\end{array}\right)}_{P_{011,002}^{300}} x_{0}^{3} .
\end{gathered}
$$

To reduce the second EK-polynomial we need

$$
\begin{aligned}
\tilde{f}_{211}= & x_{0} \tilde{f}_{111}=x_{0}^{2} f_{011}, \quad \tilde{f}_{202}=x_{0} \tilde{f}_{102}=x_{0}^{2} f_{002}, \\
\widetilde{f}_{130}= & x_{0} f_{030}, \quad \tilde{f}_{121}=x_{0} \widetilde{f}_{021}, \\
\widetilde{f}_{040}= & x_{1} f_{030}-C_{030,120} \tilde{f}_{130}-C_{030,201} \\
\tilde{f}_{211}= & x_{1}^{4}+\left(-C_{030,120}^{2}-C_{011,020} C_{030,201}+C_{030,210}\right) x_{1}^{2} x_{0}^{2} \\
& +\left(-C_{011,101} C_{030,201}-C_{030,120} C_{030,201}\right) x_{2} x_{0}^{3} \\
& +\left(-C_{011,110} C_{030,201}-C_{030,120} C_{030,210}+C_{030,300}\right) x_{1} x_{0}^{3} \\
& +\left(-C_{011,200} C_{030,201}-C_{030,120} C_{030,300}\right) x_{0}^{4} .
\end{aligned}
$$

Thus, the reduction of $S^{\mathrm{EK}}\left(f_{030}, f_{011}\right)$ is:

$$
\begin{gathered}
\underbrace{\left(\begin{array}{c}
-C_{011,020} C_{011,101}^{2}-C_{011,020}^{2} C_{030,201}+C_{011,101} C_{011,110} \\
-C_{002,020} C_{030,201}-C_{011,200}
\end{array}\right)}_{P_{030,011}^{220}} x_{1}^{2} x_{0}^{2} \\
+\underbrace{\left(\begin{array}{c}
-C_{011,101}^{3}+C_{011,101}^{2} C_{030,120}-2 C_{011,020} C_{011,101} C_{030,201} \\
-C_{002,101} C_{030,201}+C_{011,110} C_{030,201}-C_{011,101} C_{030,210}+C_{030,300}
\end{array}\right)}_{P_{030,011}^{301}} x_{2} x_{0}^{3}
\end{gathered}
$$




$$
\begin{gathered}
+\underbrace{\left(\begin{array}{c}
-C_{011,101}^{2} C_{011,110}+C_{011,101} C_{011,110} C_{030,120} \\
-C_{011,020} C_{011,110} C_{030,201}-C_{011,020} C_{011,101} C_{030,210} \\
+C_{011,101} C_{011,200}-C_{011,200} C_{030,120} \\
-C_{002,110} C_{030,201}+C_{011,020} C_{030,300}
\end{array}\right)}_{P_{030,011}^{310}} x_{1} x_{0}^{3} \\
+\underbrace{\left(\begin{array}{c}
-C_{011,101}^{2} C_{011,200}+C_{011,101} C_{011,200} C_{030,120} \\
-C_{011,020} C_{011,200} C_{030,201}-C_{011,020} C_{011,101} C_{030,300} \\
-C_{002,200} C_{030,201}-C_{011,200} C_{030,210}+C_{011,110} C_{030,300}
\end{array}\right)}_{P_{030,011}^{440}} x_{0}^{4} .
\end{gathered}
$$

In order to have a $J$-marked basis, the $J$-reduction of the EKpolynomials must be 0 so that the functor $\underline{\mathbf{M f}}_{J}$ is represented by the scheme $\mathbf{M f}_{J}=\operatorname{Spec} \mathbb{Z}[\mathbf{C}] / \mathfrak{I}_{J}$, where

$\mathfrak{I}_{J}=\left(P_{011,002}^{120}, P_{011,002}^{201}, P_{011,002}^{210}, P_{011,002}^{300}, P_{030,011}^{220}, P_{030,011}^{301}, P_{030,011}^{310}, P_{030,011}^{400}\right)$.

Now, for any ring $A$, each element of $\underline{\mathbf{M f}}{ }_{J}(A)$ is given by a scheme morphism $\operatorname{Spec} A \rightarrow \mathbf{M f}_{J}$, or equivalently by a ring morphism $\mathbb{Z}[\mathbf{C}] \rightarrow$ $A$, that factors through

$$
\mathbb{Z}[\mathbf{C}] \longrightarrow \mathbb{Z}[\mathbf{C}] / \mathfrak{I}_{J} \longrightarrow A .
$$

For instance, for $A=\mathbb{Z}[t]$, the ring morphism $\mathbb{Z}[\mathbf{C}] \rightarrow \mathbb{Z}[t]$ given by

$$
\begin{array}{lllll}
C_{002,020} & \mapsto 1-t & C_{002,101} \mapsto 0 & C_{002,110} \mapsto t^{3}-t^{4} & C_{002,200} \mapsto-t^{2} \\
C_{011,020} \mapsto 0 & C_{011,101} \mapsto 0 & C_{011,110} \mapsto t & & C_{011,200} \mapsto t^{2}-t \\
C_{030,120} \mapsto t^{3} & C_{030,201} \mapsto t & C_{030,210} \mapsto 0 & & C_{030,300} \mapsto-t^{2}
\end{array}
$$

factors through

$$
\mathbb{Z}[\mathbf{C}] \longrightarrow \mathbb{Z}[\mathbf{C}] / \mathfrak{I}_{J} \longrightarrow \mathbb{Z}[t]
$$

therefore, the following is a $J$-marked basis in $\mathbb{Z}[t]\left[x_{0}, x_{1}, x_{2}\right]$ :

$$
\begin{aligned}
& f_{002}=x_{2}^{2}+(1-t) x_{1}^{2}-\left(t^{4}-t^{3}\right) x_{1} x_{0}-t^{2} x_{0}^{2}, \\
& f_{011}=x_{2} x_{1}+t x_{1} x_{0}+\left(t^{2}-t\right) x_{0}^{2}, \\
& f_{030}=x_{1}^{3}+t^{3} x_{1}^{2} x_{0}+t x_{2} x_{0}^{2}-t^{2} x_{0}^{3} .
\end{aligned}
$$

3. Marked schemes and truncation ideals. An ideal $I \in$ $\underline{\text { Mf }}_{J}(A)$ defines a quotient algebra $A[\mathbf{x}] / I$ that is a free $A$-module, so that the family $\operatorname{Proj} A[\mathbf{x}] / I \rightarrow \operatorname{Spec} A$ is flat and defines a morphism from Spec $A$ to a suitable Hilbert scheme, by the universal property of Hilbert schemes. Thus, it is natural to study the relation between 
marked schemes and Hilbert schemes. Since Hilbert schemes parametrize flat families of subschemes of a projective space, and the same subscheme can be defined by infinitely many different ideals, we first need to investigate the function that associates the scheme it defines in $\mathbb{P}_{A}^{n}$ to every ideal in $\underline{\mathbf{M f}}_{J}(A)$. In general, this function can be noninjective, as the following example shows.

Example 3.1 (cf., [4, Example 3.4]). Consider the strongly stable ideal $J=\left(x_{2}, x_{1}^{2}, x_{1} x_{0}\right)$ in $\mathbb{Z}\left[x_{0}, x_{1}, x_{2}\right]$. For any ring $A$ and $a \in A$, consider the $J$-marked set $F_{J, a}=\left\{x_{2}+a x_{1}, x_{1}^{2}, x_{1} x_{0}\right\}$. These marked sets are, in fact, $J$-marked bases since the unique EK-polynomial involving the first generator

$$
S^{\mathrm{EK}}\left(x_{2}+a x_{1}, x_{1}^{2}\right)=x_{1}^{2}\left(x_{2}+a x_{1}\right)-x_{2}\left(x_{1}^{2}\right)=a x_{1}^{3}
$$

is clearly contained in $\left\langle F_{J, a}^{(3)}\right\rangle$. Moreover, for every $a$, the ideal $\left(F_{J, a}\right)_{\geqslant 2}$ coincides with $J_{\geq 2} \otimes A$, since

$$
\begin{aligned}
x_{2}^{2} & =x_{2}\left(x_{2}+a x_{1}\right)-a x_{1}\left(x_{2}+a x_{1}\right)+a^{2}\left(x_{1}^{2}\right), \\
x_{2} x_{1} & =x_{1}\left(x_{2}+a x_{1}\right)-a\left(x_{1}^{2}\right)
\end{aligned}
$$

and

$$
x_{2} x_{0}=x_{0}\left(x_{2}+a x_{1}\right)-a\left(x_{1} x_{0}\right) .
$$

Therefore, for all $a \in A$, the ideals $\left(F_{J, a}\right)$ define the same scheme $\operatorname{Proj} A[\mathbf{x}] / J$.

The following proposition states that non-uniqueness is a consequence of divisibility by $x_{0}$.

Proposition 3.2 (cf., [4, Theorem 3.3]). Let $J$ be a strongly stable ideal, and let $m$ be the minimum degree such that $J_{m} \neq 0$. Assume that no monomial of degree larger than $m$ in the monomial basis $\mathcal{B}_{J}$ is divisible by $x_{0}$ (or equivalently that $x_{0}^{t} \mathcal{N}(J)_{\geq m} \subseteq \mathcal{N}(J)_{\geq m+t}$ for every $t)$. Then, for any two different $J$-marked bases $F_{J}$ and $G_{J}$ in $A[\mathbf{x}]$, the schemes $\operatorname{Proj} A[\mathbf{x}] /\left(F_{J}\right)$ and $\operatorname{Proj} A[\mathbf{x}] /\left(G_{J}\right)$ are different.

Proof. By hypothesis and by Proposition 2.1 (ii), there exists a monomial $x^{\alpha} \in \mathcal{B}_{J}$ such that the corresponding polynomials $f_{\alpha} \in F_{J}$ 
and $g_{\alpha} \in G_{J}$ are different. If

$$
\operatorname{Proj} A[\mathbf{x}] /\left(F_{J}\right)=\operatorname{Proj} A[\mathbf{x}] /\left(G_{J}\right),
$$

then $\left(F_{J}\right)_{\geq s}=\left(G_{J}\right)_{\geq s}$ for a sufficiently large $s$. Therefore, for $s \gg 0$, $x_{0}^{s} f_{\alpha}$ is contained in $\left(G_{J}\right)$ and

$$
x_{0}^{s} f_{\alpha}-x_{0}^{s} g_{\alpha}=x_{0}^{s}\left(\mathrm{~T}\left(g_{\alpha}\right)-\mathrm{T}\left(f_{\alpha}\right)\right) \in\left(G_{J}\right) .
$$

By definition, the support of $\mathrm{T}\left(g_{\alpha}\right)-\mathrm{T}\left(f_{\alpha}\right)$ is contained in $\mathcal{N}(J)$. Therefore, also, the support of $x_{0}^{s}\left(\mathrm{~T}\left(g_{\alpha}\right)-\mathrm{T}\left(f_{\alpha}\right)\right)$ is in $\mathcal{N}(J)$, due to the hypothesis on $J$. Finally, by Theorem 1.7 (ii)-(vi), this implies

$$
x_{0}^{s}\left(\mathrm{~T}\left(g_{\alpha}\right)-\mathrm{T}\left(f_{\alpha}\right)\right) \in\left(F_{J}\right)_{s+|\alpha|} \cap\langle\mathcal{N}(J)\rangle=\{0\},
$$

so that $\mathrm{T}\left(g_{\alpha}\right)=\mathrm{T}\left(f_{\alpha}\right)$, against the assumption $f_{\alpha} \neq g_{\alpha}$.

Definition 3.3. We say that $J$ is an $m$-truncation ideal ( $m$-truncation for short) if $J=J_{\geq m}^{\prime}$ for $J^{\prime}$ is a saturated strongly stable ideal.

Observe that the monomials divisible by $x_{0}$ in the monomial basis of an $m$-truncation ideal $J$ (if any) are of degree $m$. Therefore, by Proposition 3.2, different $m$-truncation ideals define different projective schemes. We emphasize that a priori the truncation degree $m$ can be any positive integer. We will discuss special values of $m$ later in the paper.

We now describe the relations among marked functors (respectively, schemes) corresponding to different truncations of the same saturated strongly stable ideal $J$. We will prove that, for sufficiently large integers $m$, the $J_{\geq m}$-marked schemes are all isomorphic. However, the construction of $\mathbf{M} \mathbf{f}_{J_{\geq m}}$ given in Theorem 2.6 depends on $m$ since we obtain it as a closed subscheme of an affine space whose dimension increases with $m$. From a computational point of view it will be convenient to choose, among isomorphic marked schemes, the one corresponding to the minimum value of $m$, while for other applications higher values of $m$ can be more convenient.

In order to compare $J_{\geq m}$-marked bases in $A[\mathbf{x}]$ for different values of $m$, we refer to Proposition 3.2. By associating to a marked basis the scheme it defines, we will identify

$$
I=\left(F_{J_{\geq m}}\right) \in \underline{\mathbf{M f}}_{J_{\geq m}}
$$


and

$$
I^{\prime}=\left(G_{J_{\geq m^{\prime}}}\right) \in \underline{\mathbf{M f}}_{J_{\geq m^{\prime}}}(A)
$$

when

$$
\operatorname{Proj} A[\mathbf{x}] / I=\operatorname{Proj} A[\mathbf{x}] / I^{\prime}
$$

in $\mathbb{P}_{A}^{n}$, i.e., when $I_{\geq s}=I_{\geq s}^{\prime}$, for $s \gg 0$. By Theorem 1.7 (iii) and Proposition 2.1 (ii), this is equivalent to

$$
\widetilde{F}_{J_{\geq m}}^{(s)}=\widetilde{G}_{J_{\geq m^{\prime}}}^{(s)} \quad \text { for } s \gg 0 .
$$

Theorem 3.4. Let $J$ be a saturated strongly stable ideal. Then, for every $s>0$ and for any Noetherian ring $A, \underline{\mathbf{M f}}_{J_{\geq s-1}}(A) \subseteq \underline{\mathbf{M f}}_{J_{\geq s}}(A)$. More precisely,

(i) if $J$ has no minimal generators of degree $s+1$ divisible by the variable $x_{1}$ or $J_{\geqslant s-1}=J_{\geqslant s}$, then $\underline{\mathbf{M f}}_{J_{\geq s-1}}=\underline{\mathbf{M f}}_{J_{\geq s}}$;

(ii) otherwise, $\underline{\mathbf{M f}}_{J_{\geq s-1}}$ is a proper closed subfunctor of $\underline{\mathbf{M f}}_{J_{\geq s}}$.

Proof. To prove the inclusion $\underline{\mathbf{M f}}_{J_{\geq s-1}}(A) \subseteq \underline{\mathbf{M f}}_{J_{\geq s}}(A)$, let us consider a $J_{\geqslant s-1}$-marked basis $F$. The set

$$
G:=\widetilde{F}^{(s)} \cup\left\{f_{\alpha} \in F \mid x^{\alpha} \in \mathcal{B}_{J} \quad \text { and } \quad|\alpha|>s\right\}
$$

is, by construction, a $J_{\geqslant s}$-marked set. In fact, $G$ is a $J_{\geq s}$-marked basis, since $\left\langle G^{(s)}\right\rangle=\left\langle F^{(s)}\right\rangle$ by Theorem 1.7 (iii)-(iv), and the generators of degree larger than $s$ are the same in the two marked sets.

From now on in this proof we denote by $J^{\prime}$ the truncation of $J$ in degree $s-1$, and by $\mathcal{F}_{J^{\prime}}$ the marked set analogous to the one given in equation (2.4) that we use to construct the ideal $\mathfrak{I}_{J^{\prime}} \subseteq \mathbb{Z}\left[\mathbf{C}^{\prime}\right]$ of $\mathbf{M f}_{J^{\prime}}$. We also let

$$
A^{\prime}:=\mathbb{Z}\left[\mathbf{C}^{\prime}\right] / \mathfrak{I}_{J^{\prime}}, \quad \phi_{F_{J^{\prime}}}: \mathbb{Z}\left[\mathbf{C}^{\prime}\right] \longrightarrow A^{\prime}
$$

be the canonical map on the quotient and $\phi_{F_{J^{\prime}}}[\mathbf{x}]$ the extension to $\mathbb{Z}\left[\mathbf{C}^{\prime}\right][\mathbf{x}] \rightarrow A^{\prime}[\mathbf{x}]$. Moreover, $J^{\prime \prime}$ will be the truncation of $J$ in degree $s$ and $\mathcal{F}_{J^{\prime \prime}}, \mathbb{Z}\left[\mathbf{C}^{\prime \prime}\right], \mathfrak{I}_{J^{\prime \prime}}, A^{\prime \prime}, \phi_{F_{J^{\prime \prime}}}$ are defined analogously. By the definitions of $\mathfrak{I}_{J^{\prime}}$ and $\mathfrak{I}_{J^{\prime \prime}}$, we observe that $\phi_{F_{J^{\prime}}}[\mathbf{x}]\left(\mathcal{F}_{J^{\prime}}\right)$ is a $J^{\prime}$-marked basis in $A^{\prime}[\mathbf{x}]$ and $\phi_{F_{J^{\prime \prime}}}[\mathbf{x}]\left(\mathcal{F}_{J^{\prime \prime}}\right)$ is a $J^{\prime \prime}$-marked basis in $A^{\prime \prime}[\mathbf{x}]$. 
We first prove (ii). Let us consider the $J^{\prime \prime}$-marked set,

$$
\mathcal{G}:=\widetilde{\mathcal{F}}_{J^{\prime}}^{(s)} \cup\left\{f_{\alpha}^{\prime} \in \mathcal{F}_{J^{\prime}}\left|x^{\alpha} \in \mathcal{B}_{J},\right| \alpha \mid>s\right\} .
$$

By Theorem $1.7(\mathrm{v})-($ viii $), \phi_{F_{J^{\prime}}}[\mathbf{x}](\mathcal{G})$ is a $J^{\prime \prime}$-marked basis of $A^{\prime}[\mathbf{x}]$, since

$$
\mathcal{N}\left(J^{\prime \prime},\left(\phi_{F_{J^{\prime}}}[\mathbf{x}](\mathcal{G})\right)\right) \subseteq \mathcal{N}\left(J^{\prime},\left(\phi_{F_{J^{\prime}}}[\mathbf{x}]\left(\mathcal{F}_{J^{\prime}}\right)\right)\right)=\{0\} .
$$

Thus, the ring homomorphism

$$
\begin{aligned}
\psi: \mathbb{Z}\left[\mathbf{C}^{\prime \prime}\right] & \longrightarrow \mathbb{Z}\left[\mathbf{C}^{\prime}\right] \\
C_{\alpha \beta}^{\prime \prime} & \longmapsto \text { coefficient of } x^{\beta} \text { in } g_{\alpha} \in \mathcal{G}
\end{aligned}
$$

induces a homomorphism $\bar{\psi}: A^{\prime \prime} \rightarrow A^{\prime}$ such that $\phi_{F_{J^{\prime}}} \circ \psi=\bar{\psi} \circ \phi_{F_{J^{\prime \prime}}}$. Moreover, $\phi_{F_{J^{\prime}}} \circ \psi$ is surjective, being the composition of two surjective homomorphisms. Indeed,

$$
C_{\alpha \beta}^{\prime}= \begin{cases}\psi\left(C_{\alpha \beta}^{\prime \prime}\right), & \text { if } x^{\alpha} \in \mathcal{B}_{J},|\alpha| \geq s, \\ \psi\left(C_{\eta \gamma}^{\prime \prime}\right), x^{\eta}=x_{0} x^{\alpha}, x^{\gamma}=x_{0} x^{\beta}, & \text { otherwise. }\end{cases}
$$

Under our assumptions, for every $f_{\alpha}^{\prime} \in \mathcal{F}_{J^{\prime}}$ of degree $s-1, x_{0} T\left(f_{\alpha}^{\prime}\right)$ is a $J^{\prime \prime}$-remainder, so that $x_{0} f_{\alpha}^{\prime} \in \mathcal{G}$.

Therefore, the epimorphism $\bar{\psi}$ induces an isomorphism between the marked scheme $\mathbf{M f}_{J^{\prime}}=\operatorname{Spec} \mathbb{Z}\left[\mathbf{C}^{\prime}\right] / \mathfrak{I}_{J^{\prime}}$ and a closed subscheme of $\mathbf{M f}_{J^{\prime \prime}}=\operatorname{Spec} \mathbb{Z}\left[\mathbf{C}^{\prime \prime}\right] / \mathfrak{I}_{J^{\prime \prime}}$. In order to show that this subscheme is proper we can look at the Zariski tangent spaces of $\mathbf{M f}_{J^{\prime}}$ and $\mathbf{M} \mathbf{f}_{J^{\prime \prime}}$ at the points corresponding to $J^{\prime}$ and $J^{\prime \prime}$ and see that they have different dimension (see [4, Theorem 5.7] for the details).

To prove (i), we observe that the new condition on $J$ implies that, for every $x^{\gamma} \in \mathcal{N}(J)_{s}$, either $x_{1} x^{\gamma} \in \mathcal{N}(J)_{s+1}$ or $x_{1} x^{\gamma}=x_{0} x^{\delta}$ with $x^{\delta} \in J_{s}$ holds.

Exploiting this property, we first prove that $C_{\eta \gamma}^{\prime \prime} \in \mathfrak{I}_{J^{\prime \prime}}$ if $x^{\eta} \in J_{s}$, $x_{0} \mid x^{\eta}$ and $x_{0} \nmid x^{\gamma}$. Let $x^{\epsilon}=x_{1} x^{\eta} / x_{0}$, and consider the EK-syzygy $S^{\mathrm{EK}}\left(f_{\eta}^{\prime \prime} f_{\epsilon}^{\prime \prime}\right)=x_{0} T\left(f_{\epsilon}^{\prime \prime}\right)-x_{1} T\left(f_{\eta}^{\prime \prime}\right)$ between the elements $f_{\eta}^{\prime \prime}, f_{\epsilon}^{\prime \prime} \in \mathcal{F}_{J^{\prime \prime}}$.

The $J$-remainder of this polynomial, given by $\stackrel{\mathcal{F}_{J^{\prime \prime}}^{(\cdot)}}{\longrightarrow}$, is of the type

$$
g=x_{0} T\left(f_{\epsilon}^{\prime \prime}\right)-x_{1} T\left(f_{\eta}^{\prime \prime}\right)+x_{0} \sum C_{\eta \delta}^{\prime \prime} f_{\beta}^{\prime \prime},
$$


where $f_{\beta}^{\prime \prime} \in \mathcal{F}_{J^{\prime \prime}}$, and the sum is over the multi-indices $\beta$ such that $x^{\beta}:=x_{1} x^{\delta} / x_{0} \in J_{s}$ with $x^{\delta}$ divisible by $x_{0}$ and contained in the support of $T\left(f_{\eta}^{\prime \prime}\right)$, and $C_{\eta \delta}^{\prime \prime}$ the coefficient of $x^{\delta}$ in $f_{\eta}^{\prime \prime}$. If $x^{\gamma}$ is a term in the support of $T\left(f_{\eta}^{\prime \prime}\right)$ such that $x_{0} \nmid x^{\gamma}$, then $x_{1} x^{\gamma} \in \mathcal{N}(J)_{s+1}$ is contained in the support of $g$. By definition, $\mathfrak{I}_{J^{\prime \prime}}$ contains the $\mathbf{x}$ coefficients of $g$, thus, in particular, the coefficient $C_{\eta \gamma}^{\prime \prime}$ of $x_{1} x^{\gamma}$ in $g$. For every $x^{\alpha} \in J_{s-1}$ and $x^{\eta}=x_{0} x^{\alpha}$, let us denote by $h_{\alpha}$ the polynomial in $\mathbb{Z}\left[\mathbf{C}^{\prime \prime}\right][\mathbf{x}]$ such that

$$
f_{\eta}^{\prime \prime}=x_{0} h_{\alpha}+\sum C_{\eta \gamma}^{\prime \prime} x^{\gamma}
$$

with $x_{0} \nmid x^{\gamma}$, so that $\phi_{F_{J^{\prime \prime}}}[\mathbf{x}]\left(f_{\eta}^{\prime \prime}\right)=\phi_{F_{J^{\prime \prime}}}[\mathbf{x}]\left(x_{0} h_{\alpha}\right)$.

Using these polynomials, we can define the $J^{\prime}$-marked set

$$
\mathcal{H}=\left\{h_{\alpha} \mid x^{\alpha} \in J_{s-1}\right\} \cup\left\{f_{\eta}^{\prime \prime} \in \mathcal{F}_{J^{\prime \prime}}\left|x^{\eta} \in \mathcal{B}_{J},\right| \eta \mid \geq s\right\} .
$$

By construction,

$$
\phi_{F_{J^{\prime \prime}}}[\mathbf{x}]\left(x_{0} \mathcal{H}\right) \subseteq \phi_{F_{J^{\prime \prime}}}[\mathbf{x}]\left(\mathcal{F}_{J^{\prime \prime}}\right)
$$

therefore, $\phi_{F_{J^{\prime \prime}}}[\mathbf{x}](\mathcal{H})$ is a $J^{\prime}$-marked basis by Theorem $1.7(\mathrm{v})$-(viii). In fact, if the support of an element $u$ in the ideal $A^{\prime \prime}\left(\phi_{F_{J^{\prime \prime}}}[\mathbf{x}](\mathcal{H})\right)$ only contains monomials of $\mathcal{N}(J)$, then $x_{0} u$ has the same support and is in $A^{\prime \prime}\left(\phi_{F_{J^{\prime \prime}}}[\mathbf{x}]\left(\mathcal{F}_{J^{\prime \prime}}\right)\right)$, so that $u=0$ since $\mathcal{N}\left(J,\left(\phi_{F_{J^{\prime \prime}}}[\mathbf{x}]\left(\mathcal{F}_{J^{\prime \prime}}\right)\right)\right)=\{0\}$.

Thus, the ring homomorphism,

$$
\begin{aligned}
\varphi: \mathbb{Z}\left[\mathbf{C}^{\prime}\right] & \longrightarrow \mathbb{Z}\left[\mathbf{C}^{\prime \prime}\right] \\
C_{\alpha \beta}^{\prime} & \longmapsto \text { coefficient of } x^{\beta} \text { in } h_{\alpha} \text { if }|\alpha|=s-1 \\
C_{\eta \gamma}^{\prime} & \longmapsto \text { coefficient of } x^{\gamma} \text { in } f_{\eta}^{\prime \prime} \text { if } x^{\eta} \in \mathcal{B}_{J},|\eta| \geq s,
\end{aligned}
$$

induces a homomorphism $\bar{\varphi}: A^{\prime} \rightarrow A^{\prime \prime}$.

Finally, $\bar{\psi}$ and $\bar{\varphi}$ are inverses of each other. Indeed, if we apply the construction from the first part of the proof to the $J^{\prime}$-marked set $\mathcal{H}$, we obtain a $J^{\prime \prime}$-marked set $\mathcal{G}^{\prime}$ such that $\phi_{F_{J^{\prime \prime}}}[\mathbf{x}]\left(\mathcal{G}^{\prime}\right)$ is a $J^{\prime \prime}$-marked basis and $\phi_{F_{J^{\prime \prime}}}[\mathbf{x}]\left(\mathcal{G}^{\prime}\right) \subseteq \phi_{F_{J^{\prime \prime}}}[\mathbf{x}]\left(\mathcal{F}_{J^{\prime \prime}}\right)$; hence, $\phi_{F_{J^{\prime \prime}}}[\mathbf{x}]\left(\mathcal{G}^{\prime}\right)=\phi_{F_{J^{\prime \prime}}}[\mathbf{x}]\left(\mathcal{F}_{J^{\prime \prime}}\right)$ by Proposition 2.1.

4. Marked and Hilbert schemes. We now briefly recall how the Hilbert scheme can be constructed as a subscheme of a suitable Grassmannian. For any positive integer $n$ and any numerical polynomial 
$p(t)$, consider the Hilbert functor

$$
\underline{\text { Hilb }}_{n}^{p(t)}: \underline{\text { Noeth-Rings }} \longrightarrow \underline{\text { Sets }}
$$

associated to any Noetherian ring $A$ the set

$$
\underline{\mathbf{H i l b}}_{n}^{p(t)}(A)=\left\{X \subseteq \mathbb{P}_{A}^{n} \mid \begin{array}{c}
X \rightarrow \operatorname{Spec} A \text { is flat and } \\
\text { has fibers with Hilbert polynomial } p(t)
\end{array}\right\}
$$

and to any ring homomorphism $f: A \rightarrow B$ the map

$$
\begin{aligned}
\underline{\mathbf{H i l b}}_{n}^{p(t)}(f): \underline{\mathbf{H i l b}}_{n}^{p(t)}(A) & \longrightarrow \underline{\mathbf{H i l b}}_{n}^{p(t)}(B) \\
X & \longmapsto X \times_{\operatorname{Spec} A} \operatorname{Spec} B .
\end{aligned}
$$

Grothendieck first defined this functor and showed that it is representable [15]. The Hilbert scheme Hilb ${ }_{n}^{p(t)}$ is defined as the scheme representing the Hilbert functor, and it is classically constructed as a subscheme of a suitable Grassmannian. Let us briefly recall how (for a detailed exposition, see $[\mathbf{6}, \mathbf{1 6}, \mathbf{1 8}])$. By Gotzmann's regularity theorem ([13, equation (2.9)] and [18, Lemma C.23]), there exists a positive integer $r$ depending only on $p(t)$, called the Gotzmann number, for which the ideal sheaf $\mathcal{I}_{X}$ of each scheme $X \in \underline{\mathbf{H i l b}}_{n}^{p(t)}(A)$ is $r$-regular (in the sense of Castelnuovo-Mumford regularity). This implies that the morphism

$$
H^{0}\left(\mathcal{O}_{\mathbb{P}_{A}^{n}}(r)\right) \stackrel{\phi_{X}}{\longrightarrow} H^{0}\left(\mathcal{O}_{X}(r)\right)
$$

is surjective. By flatness, $H^{0}\left(\mathcal{O}_{X}(r)\right)$ is a locally free module of rank $p(r)$ and, as an $A$-module, $H^{0}\left(\mathcal{O}_{\mathbb{P}_{A}^{n}}(r)\right)$ is isomorphic to the homogeneous piece of degree $r$ of the polynomial ring $A[\mathbf{x}]$. Since $A[\mathbf{x}]_{r} \simeq A^{N}$, where $N=\left(\begin{array}{c}n+r \\ n\end{array}\right)$, the homomorphism $\phi_{X}$ may be viewed as an element of the Grassmannian, whose corresponding functor is

$$
\underline{\mathbf{G r}}_{p(r)}^{N}: \underline{\text { Noeth-Rings }} \longrightarrow \underline{\text { Sets}},
$$

associating to any Noetherian ring $A$ the set

$$
\underline{\mathbf{G r}}_{p(r)}^{N}(A)=\left\{\begin{array}{c}
\text { isomorphism classes of epimorphisms } A^{N} \longrightarrow Q \\
\text { of locally free modules of rank } p(r)
\end{array}\right\}
$$

and to any morphism $f: A \rightarrow B$, the map

$$
\begin{aligned}
\underline{\mathbf{G r}}_{p(r)}^{N}(f): \underline{\mathbf{G r}}_{p(r)}^{N}(A) & \longrightarrow \underline{\mathbf{G r}}_{p(r)}^{N}(B) \\
A^{N} \longrightarrow Q & \longmapsto B^{N} \longrightarrow Q \otimes_{A} B .
\end{aligned}
$$


Two epimorphisms $\phi: A^{N} \rightarrow Q$ and $\phi^{\prime}: A^{N} \rightarrow Q^{\prime}$ are isomorphic if there exists an isomorphism $\psi: Q \rightarrow Q^{\prime}$ of $A$-modules such that the diagram

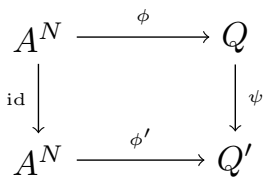

commutes. Equivalently, $\phi$ and $\phi^{\prime}$ are isomorphic if $\operatorname{ker} \phi=\operatorname{ker} \phi^{\prime}$. Therefore, by identifying isomorphism classes of epimorphisms $\phi$ with ker $\phi$, the Grassmann functor sends $A$ to the set

$$
\left\{\begin{array}{l}
A \text {-submodules } M \subseteq A^{N} \text { such that } \\
A^{N} / M \text { is locally free of } \operatorname{rank} p(r)
\end{array}\right\} .
$$

This functor is representable, and the representing scheme $\mathbf{G r}_{p(r)}^{N}$ is the Grassmannian (see [28, subsection 16.7]). Therefore, one of the possible embeddings of the Hilbert scheme into a Grassmannian is given by the natural transformation of functors (introduced by Bayer [3])

$$
\underline{\mathscr{H}}: \underline{\mathbf{H i l b}}_{n}^{p(t)} \longrightarrow \underline{\mathbf{G r}}_{p(r)}^{N}
$$

given by

$$
\begin{aligned}
\underline{\mathbf{H i l b}}_{n}^{p(t)}(A) & \longrightarrow \underline{\mathbf{G r}}_{p(r)}^{N}(A) \\
X & \longmapsto A[\mathbf{x}]_{r} \rightarrow H^{0}\left(\mathcal{O}_{X}(r)\right) .
\end{aligned}
$$

By Yoneda's lemma, any natural transformation of representable functors is induced by a unique morphism between their representing schemes. The associated morphism $\mathscr{H}: \mathbf{H i l b}_{n}^{p(t)} \rightarrow \mathbf{G r}_{p(r)}^{N}$ is a closed embedding, and the equations defining the Hilbert scheme $\mathbf{H i l b}_{n}^{p(t)}$ as a subscheme of $\mathbf{G r}_{p(r)}^{N}$ were conjectured by Bayer [3] and proved much later by Haiman and Sturmfels [16].

The Grassmannian has the well-known open cover by affine spaces which also defines the Plücker embedding. For any set $\mathcal{N}$ of $p(r)$ distinct monomials of $A[\mathbf{x}]_{r}$, consider the map,

$$
\mathrm{i}_{\mathcal{N}}:{ }_{A}\langle\mathcal{N}\rangle \simeq A^{p(r)} \hookrightarrow A[\mathbf{x}]_{r} \simeq A^{N}
$$


and the subfunctor $\underline{\mathbf{G}}_{\mathcal{N}}$ such that

$$
\underline{\mathbf{G}}_{\mathcal{N}}(A)=\left\{\begin{array}{c}
\text { classes } \phi_{Q}: A^{N} \longrightarrow Q \text { in } \mathbf{G r}_{p(r)}^{N}(A) \\
\text { such that } \phi_{Q} \circ \mathrm{i}_{\mathcal{N}} \text { is surjective }
\end{array}\right\} .
$$

Each such subfunctor is open, and the family obtained varying the set of monomials $\mathcal{N}$ covers the Grassmann functor [27, Lemma 26.22.1]. Since $\phi_{Q} \circ i_{\mathcal{N}}$ is an epimorphism between a free module and a locally free module of the same rank, it is in fact an isomorphism. Therefore, each $Q$ in $\underline{\mathbf{G}}_{\mathcal{N}}(A)$ can be identified with the free module ${ }_{A}\langle\mathcal{N}\rangle$, and we can rewrite the functors $\underline{\mathbf{G}}_{\mathcal{N}}$ as

$$
\underline{\mathbf{G}}_{\mathcal{N}}(A)=\left\{\begin{array}{c}
\text { epimorphisms } A[\mathbf{x}]_{r} \longrightarrow{ }_{A}\langle\mathcal{N}\rangle \\
\text { of free modules of } \operatorname{rank} p(r)
\end{array}\right\} .
$$

An epimorphism $\phi: A[\mathbf{x}]_{r} \rightarrow{ }_{A}\langle\mathcal{N}\rangle$ is determined by its values on basis elements,

$$
\phi\left(x^{\alpha}\right)=\sum_{x^{\beta} \in \mathcal{N}} a_{\alpha, \beta} x^{\beta} .
$$

Thus, its kernel is generated by

$$
f_{\alpha}:=x^{\alpha}-\sum_{x^{\beta} \in \mathcal{N}} a_{\alpha \beta} x^{\beta},
$$

for all $x^{\alpha}$ of total degree $r$ lying outside $\mathcal{N}$. If $J$ is the ideal generated by the monomials in $A[\mathbf{x}]_{r}$ not contained in $\mathcal{N}$, then we can describe $\underline{\mathbf{G}}_{\mathcal{N}}$ as

$$
\begin{aligned}
\underline{\mathbf{G}}_{\mathcal{N}}(A) & =\left\{\text { free submodules } L \subseteq A[\mathbf{x}]_{r} \text { such that } A[\mathbf{x}]_{r} \simeq L \oplus_{A}\langle\mathcal{N}\rangle\right\} \\
& =\left\{\text { submodules } L \subseteq A[\mathbf{x}]_{r} \text { generated by a } J \text {-marked set }\right\}
\end{aligned}
$$

We are interested in the open subfunctors of the Hilbert functor $\underline{\text { Hilb }}_{n}^{p(t)}$ induced by the family of subfunctors $\underline{\mathbf{G}}_{\mathcal{N}}$ by means of $\underline{\mathscr{H}}$. We denote by $\underline{\mathbf{H}}_{\mathcal{N}}$ the subfunctor associating to $A$ the set

$$
\underline{\mathbf{H}}_{\mathcal{N}}(A):=\underline{\mathscr{H}}^{-1}\left(\underline{\mathbf{G}}_{\mathcal{N}}(A) \cap \underline{\mathscr{H}}\left(\underline{\mathbf{H i l b}}_{n}^{p(t)}(A)\right)\right) .
$$

The kernel of the map $A[\mathbf{x}]_{r} \rightarrow H^{0}\left(\mathcal{O}_{X}(r)\right)$ is represented by the global sections of the sheaf $\mathcal{I}_{X}(r)$, i.e., by the homogeneous piece of degree $r$ of the saturated ideal $I_{X}$ defining $X$. Since $I_{X}$ and $\left(I_{X}\right)_{\geqslant r}$ define the same scheme and $\left(I_{X}\right)_{\geqslant r}$ is generated by the homogeneous 
piece of degree $r$, we can rewrite the subfunctor $\underline{\mathbf{H}}_{\mathcal{N}}$ as

$$
\begin{aligned}
\underline{\mathbf{H}}_{\mathcal{N}}(A) & =\left\{X \in \underline{\mathbf{H i l b}}_{n}^{p(t)}(A) \mid A[\mathbf{x}]_{r} \simeq H^{0}\left(\mathcal{I}_{X}(r)\right) \oplus{ }_{A}\langle\mathcal{N}\rangle\right\} \\
& =\left\{X \in \underline{\operatorname{Hilb}}_{n}^{p(t)}(A) \mid \begin{array}{c}
\left(I_{X}\right)_{\geqslant r} \text { is generated by } \\
\text { a } J \text {-marked set }
\end{array}\right\} .
\end{aligned}
$$

It is then natural to relate $\underline{\mathbf{H}}_{\mathcal{N}}(A)$ to $\underline{\mathbf{M f}}_{J}(A)$. In general, their relations are less obvious than one might expect. However, under suitable conditions on $\mathcal{N}$ and $J$, we can identify $\underline{\mathbf{H}}_{\mathcal{N}}$ with a marked functor. The following result gives a new proof in terms of functors of $[5$, Theorem 2.5].

Lemma 4.1. Let $p(t)$ be a Hilbert polynomial in $\mathbb{P}^{n}$ with Gotzmann number $r$, and let $J$ be a strongly stable ideal such that $\left|\mathcal{N}(J)_{r}\right|=p(r)$. Then, for every Noetherian ring A,

$$
\underline{\mathbf{H}}_{\mathcal{N}(J)_{r}}(A) \neq \emptyset \Longleftrightarrow \text { the Hilbert polynomial of } A[\mathbf{x}] / J \text { is } p(t) \text {. }
$$

\section{Proof.}

$(\Leftarrow)$. If the Hilbert polynomial of $A[\mathbf{x}] / J$ is $p(t)$, then $\operatorname{Proj} A[\mathbf{x}] / J \in$ $\underline{\mathbf{H}}_{\mathcal{N}}(A)$.

$(\Rightarrow)$. Assume that $X$ is a scheme in $\underline{\mathbf{H}}_{\mathcal{N}}(A)$, and set $I:=\left(I_{X}\right)_{\geqslant r}$. By Theorem 1.7 (iii), for every $m \geq r$, the $A$-module $I_{m}$ has a free direct summand with rank equal to that of $J_{m}$; therefore, the value of the Hilbert polynomial of $J$ in every degree $m \geq r$ cannot be smaller than $p(m)$. On the other hand, this rank cannot be larger than $p(m)$ by Macaulay's estimate on the growth of ideals [14, Theorem 3.3].

Corollary 4.2. Let $J$ be a saturated strongly stable ideal such that $\mathbb{Z}[\mathbf{x}] / J$ has Hilbert polynomial $p(t)$. Then

$$
\underline{\mathbf{H}}_{\mathcal{N}(J)_{r}} \simeq \underline{\mathbf{M f}}_{J_{\geq r}} .
$$

We can rephrase the statement of the corollary by saying that, for every Noetherian ring $A$,

$$
\underline{\mathbf{H}}_{\mathcal{N}(J)_{r}}(A)=\left\{\begin{array}{l|c}
X \in \underline{\mathbf{H i l b}}_{n}^{p(t)}(A) & \begin{array}{c}
\left(I_{X}\right)_{\geqslant r} \text { is generated by } \\
\text { a } J \text {-marked basis }
\end{array}
\end{array}\right\} .
$$


Therefore, upon identifying ideals and the schemes they define, the isomorphism from Corollary 4.2, is a canonical identification $\underline{\mathbf{H}}_{\mathcal{N}(J)_{r}}=$ $\underline{\mathbf{M f}}_{J_{\geq r}}$.

We can then deduce from Corollary 4.2 an isomorphism between the representing schemes. Taking into account Theorem 3.4 we obtain the following result.

Corollary 4.3. Let $J$ be a saturated strongly stable ideal, and let $r$ be the Gotzmann number of its Hilbert polynomial $p(t)$. If $\rho$ is the maximum degree of monomials in $\mathcal{B}_{J}$ divisible by $x_{1}$, then

(i) for $s \geqslant \rho-1, \mathbf{M f}_{J_{\geq s}}$ is an open subscheme of $\mathbf{H i l b}_{n}^{p(t)}$;

(ii) for $s<\rho-1, \mathbf{M f}_{J_{\geq s}}$ is a locally closed subscheme of $\mathbf{H i l b}_{n}^{p(t)}$.

Proof.

(i) By Theorem 3.4, we have

$$
\mathbf{M f}_{J_{\geq \rho-1}} \simeq \mathbf{M f}_{J_{\geq \rho}} \simeq \cdots \simeq \mathbf{M f}_{J_{\geq r}}=\mathbf{H}_{\mathcal{N}(J)_{r}} .
$$

(ii) By Theorem 3.4, for $s<\rho-1$, we know that in the chain

$$
\mathbf{M f}_{J_{\geq s}} \subseteq \mathbf{M} \mathbf{f}_{J_{\geq s+1}} \subseteq \cdots \subseteq \mathbf{M} \mathbf{f}_{J_{\geq \rho-1}} \simeq \cdots \simeq \mathbf{M f}_{J_{\geq r}}=\mathbf{H}_{\mathcal{N}(J)_{r}},
$$

there is at least one proper closed embedding, so that $\mathbf{M f}_{J_{\geq s}}$ is a locally closed subscheme of the Hilbert scheme.

Remark 4.4. Although our results only apply to a small number of the open subsets $\underline{\mathbf{H}}_{\mathcal{N}}$ necessary to cover $\mathbf{H i l b}{ }_{n}^{p(t)}$, in many interesting cases, a different open cover is obtained by exploiting the action of the linear group $\operatorname{PGL}(n+1)$. In particular, this is true for the Hilbert scheme $\operatorname{Hilb}_{n, K}^{p(t)}=\mathbf{H i l b}_{n}^{p(t)} \times_{\operatorname{Spec} \mathbb{Z}} \operatorname{Spec} K$, representing the Hilbert functor,

$$
\underline{\text { Hilb }}_{n, K}^{p(t)}: \underline{K \text {-Algebras }} \longrightarrow \underline{\text { Sets }},
$$

for every field $K$ of characteristic 0 . Indeed, the properties of the generic initial ideal proved by Galligo [12] allow us to prove that every point of the Hilbert scheme is contained in an open subset $\mathbf{H}_{\mathcal{N}, K}$, where $\mathcal{N}:=\mathcal{N}(J)_{r}$, for a saturated strongly stable ideal $J$, at least up to the action of a general element in $\operatorname{PGL}(n+1)$. Such an open cover of $\mathbf{H i l b}_{n, K}^{p(t)}$ is presented in $[\mathbf{2}, \mathbf{5}, \mathbf{6}]$. 
The set of strongly stable ideals necessary to obtain such a new open cover of the Hilbert scheme can be effectively determined using the algorithm presented in $[\mathbf{8}, \mathbf{2 0}, \mathbf{2 1}]$.

Remark 4.5. The equations of the open subscheme $\mathbf{H}_{\mathcal{N}(J)_{r}}$ computed as the marked scheme over $J_{\geq r}$, are the same as those determined by Iarrobino and Kleiman in [18]. Indeed, the Eliahou and Kervaire syzygies among the generators of $J_{\geq r}$ are linear, so that imposing

$$
S^{\mathrm{EK}}\left(f_{\alpha}, f_{\beta}\right) \stackrel{F_{J \geq r}^{(\cdot)}}{\longrightarrow} 0,
$$

is equivalent to proving that $\left\langle S F_{J_{\geq r}}^{(r+1)}\right\rangle \subseteq\left\langle F_{J_{\geq r}}^{(r+1)}\right\rangle$. If we represent the generators

$$
\left\{x_{i} f_{\alpha} \mid \text { for all } x^{\alpha} \in \mathcal{B}_{J_{\geq r}}, i=0, \ldots, n\right\}
$$

of $\left(F_{J}\right)_{r+1}$ by a matrix $\mathcal{M}_{J}^{(r+1)}$, then condition $\left\langle S F_{J_{\geq r}}^{(r+1)}\right\rangle \subseteq\left\langle F_{J_{\geq r}}^{(r+1)}\right\rangle$ is equivalent to

$$
\operatorname{rk} \mathcal{M}_{J}^{(r+1)} \leqslant \operatorname{rk}\left\langle F_{J_{\geq r}}^{(r+1)}\right\rangle=\operatorname{rk} J_{r+1}=\left(\begin{array}{c}
n+r+1 \\
n
\end{array}\right)-p(r+1),
$$

and the latter condition is guaranteed by imposing the vanishing of the minors of order rk $J_{r+1}+1$. This is how Iarrobino and Kleiman determined local equations of the Hilbert scheme. Notice that using this approach it is possible to deduce that the equations are of degree at most $\left(\begin{array}{c}n+r+1 \\ n\end{array}\right)-p(r+1)+1$, while constructing the equations applying Theorem 2.11 (ii) and our reduction procedure, it is possible to deduce that the equations have degree at most $\operatorname{deg} p(t)+2$ (see $[\mathbf{5}$, Theorem $3.3]$ ).

Remark 4.6. The statements of Corollary 4.3 can be very useful both from a computational and a theoretical point of view. Indeed, for a fixed saturated ideal $J$, the number of variables involved in the computation of equations defining the marked scheme $\mathbf{M f _ { J \geq s }}$ dramatically increases with $s$. On the other hand, in [2], the equalities of Corollary 4.3 (i) show that the open subset of $\mathbf{H i l b}_{n, K}^{p(t)}$ of the $r^{\prime}$ regular points, for a given $r^{\prime}<r$, can be embedded as a locally closed subscheme in the Grassmannian $\mathbf{G r}_{p\left(r^{\prime}\right)}^{N\left(r^{\prime}\right)}$, smaller than that in which we can embed the entire Hilbert scheme. 
Moreover, in several cases, marked schemes $\mathbf{M f}_{J_{\geq s}}$ with $s<\rho-1$ correspond to interesting loci of the Hilbert scheme, and our results also allow effective computations on them.

Example 4.7. Consider the strongly stable ideal

$$
J=\left(x_{2}^{2}, x_{2} x_{1}, x_{1}^{4}\right) \subseteq \mathbb{Z}\left[x_{0}, x_{1}, x_{2}\right] .
$$

The Hilbert polynomial of Proj $\mathbb{Z}\left[x_{0}, x_{1}, x_{2}\right] / J$ is $p(t)=5$ with Gotzmann number equal to 5 . Therefore, the open subscheme $\mathbf{H}_{\mathcal{N}(J)_{5}} \subseteq$ $\mathbf{H i l b}_{2}^{5}$ can be defined as a closed subscheme of the affine open subscheme $\mathbf{G}_{\mathcal{N}(J)_{5}} \subseteq \mathbf{G r}_{5}^{21}$ of dimension 80. Applying Corollary 4.3 (i), we can define the same open subscheme by means of the isomorphism $\mathbf{M f}_{J_{\geq 3}} \simeq \mathbf{H}_{\mathcal{N}(J)_{5}}$ with $\mathbf{M f}_{J_{\geq 3}} \subseteq \mathbb{A}^{30}$.

Finally, also the marked scheme associated to the saturated ideal may be very important. For instance, in the special case of zerodimensional schemes in the projective plane $\mathbb{P}^{2}$, for each postulation there is a unique strongly stable ideal $J$ realizing it (see for instance [10, Chapters 1-3]). Therefore, $\mathbf{M f}_{J}$ parametrizes the locus of the Hilbert scheme $\mathbf{H i l b}_{2}^{d}$ with a fixed Hilbert function (up to the action of the projective linear group). In the example, the scheme $\mathbf{M f}_{J}$ parameterizes the locus of five points in the plane with postulation $(1,3,4,5, \ldots)$.

5. Gröbner strata. Throughout this section, we denote by $\sigma$ a term ordering on the polynomial ring $A[\mathbf{x}]$ and by $\operatorname{in}_{\sigma}(I)$ the initial ideal of an ideal $I \subseteq A[\mathbf{x}]$ with respect to such term ordering. We define the Gröbner functor $\underline{\mathbf{S t}}_{J}^{\sigma}:$ Noeth-Rings $\rightarrow$ Sets that associates to any ring $A$ the set

$$
\underline{\mathbf{S t}}_{J}^{\sigma}(A)=\left\{\text { ideals } I \subseteq A[\mathbf{x}] \mid \operatorname{in}_{\sigma}(I)=J\right\},
$$

and to any ring homomorphism $\phi: A \rightarrow B$ the function

$$
\begin{aligned}
\underline{\mathbf{S t}}_{J}^{\sigma}(\phi): \underline{\mathbf{S t}}_{J}^{\sigma}(A) & \longrightarrow \underline{\mathbf{S t}}_{J}^{\sigma}(B) \\
I & \longmapsto I \otimes_{A} B .
\end{aligned}
$$

Gröbner basis theory over rings is more intricate than Gröbner basis theory over fields (see also [19] for a more detailed discussion). The first complex issue is the definition of initial ideals. Given an ideal $I \subseteq A[\mathbf{x}]$, 
we can consider the ideal generated by the leading monomials or the ideal generated by leading terms, i.e., monomials with coefficients, of the polynomials in $I$. In general, neither of the two definitions is well suited for functorial constructions, since taking the initial ideal of a given $I \subseteq A[\mathbf{x}]$ does not commute with base change $\otimes_{A} B$ unless the initial ideal of $I$ is a monomial ideal. For instance, the initial ideal of

$$
I=\left(2 x_{1}+x_{0}\right) \subseteq \mathbb{Z}\left[x_{0}, x_{1}\right], \quad x_{1}>x_{0},
$$

is $J^{\prime}=\left(x_{1}\right)$ according to the first definition and $J^{\prime \prime}=\left(2 x_{1}\right)$ according to the second one; after the extension $\mathbb{Z} \rightarrow \mathbb{Z}_{2}:=\mathbb{Z} / 2 \mathbb{Z}$ we obtain $\operatorname{in}_{\sigma}\left(I \otimes_{\mathbb{Z}} \mathbb{Z}_{2}\right)=\left(x_{0}\right)$, while $J^{\prime} \otimes_{\mathbb{Z}} \mathbb{Z}_{2}=\left(x_{1}\right)$ and $J^{\prime \prime} \otimes_{\mathbb{Z}} \mathbb{Z}_{2}=(0)$.

Definition $5.1([25,29])$. Let $I$ be an ideal in a polynomial ring $A[\mathbf{x}]$, with $A$ a Noetherian ring, and let $\sigma$ be a term ordering. The ideal $I$ is called monic (with respect to $\sigma$ ) if, for all monomials $x^{\alpha} \in A[\mathbf{x}]$, the set

$$
\mathrm{LC}\left(I, x^{\alpha}\right)=\left\{a \in A \mid a x^{\alpha} \text { is the leading term of } g \in I\right\} \cup\{0\},
$$

is either $\{0\}$ or $A$.

Therefore, the definition of $\mathbf{S t}_{J}^{\sigma}$ given in equation (5.1) is correct and non-ambiguous if we assume that $J$ is a monomial ideal and restrict the set of ideals $I$ to those that are monic. To this aim, we follow the line of the definition of marked functor and consider the ideals $I$ that are generated by a suitable set of polynomials, marked on $J$, that we expect to form a reduced Gröbner basis. Indeed, an ideal $I \subseteq A[\mathbf{x}]$ admits a reduced Gröbner basis if, and only if, $I$ is a monic ideal (see $[\mathbf{1}, \mathbf{2 5}, \mathbf{2 9}])$. We recall that a reduced Gröbner basis is a Gröbner basis composed of polynomials with leading coefficient equal to $1_{A}$ and such that no term other than the leading one is contained in the initial ideal. More precisely, the reduced Gröbner basis takes the shape

$$
G_{J}=\left\{x^{\alpha}-\sum_{\substack{x^{\beta} \in \mathcal{N}(J)|\alpha| \\ x^{\alpha}>_{\sigma} x^{\beta}}} b_{\alpha \beta} x^{\beta} \in A[\mathbf{x}] \mid x^{\alpha} \in \mathcal{B}_{J}\right\} .
$$

This is a $J$-marked set, considering the marking given by the term ordering, i.e., $\operatorname{Ht}(g)=\operatorname{in}_{\sigma}(g)$. Furthermore, $G_{J}$ is a $J$-marked basis since, for $I=\left(G_{J}\right) \in \underline{\mathbf{S t}}_{J}^{\sigma}(A)$, the monomials in $\mathcal{N}\left(\operatorname{in}_{\sigma}(I)\right)=\mathcal{N}(J)$ are 
a basis of the $A$-module $A[\mathbf{x}] / I$. Then we can rewrite $\underline{\mathbf{S t}}_{J}^{\sigma}(A)$ as

$$
\begin{aligned}
& \underline{\mathbf{S t}}_{J}^{\sigma}(A)=\left\{\text { monic ideals } I \subseteq A[\mathbf{x}] \mid \operatorname{in}_{\sigma}(I)=J\right\} \\
& =\left\{\begin{array}{l|l}
I=\left(G_{J}\right) & \begin{array}{c}
G_{J} \text { reduced Gröbner basis } \\
\text { and } \operatorname{in}_{\sigma}\left(\left(G_{J}\right)\right)=J
\end{array}
\end{array}\right\} .
\end{aligned}
$$

Thus, $\underline{\mathbf{S t}}_{J}^{\sigma}(A) \subseteq \underline{\mathbf{M f}}_{J}(A)$ for every $A$, and there is an injection of functors $\underline{\mathbf{S t}}_{J}^{\sigma} \rightarrow \underline{\mathbf{M f}}_{J}$.

Lemma 5.2. Let $J$ be any monomial ideal and $\sigma$ a term ordering. Then $\underline{\mathbf{S t}}_{J}^{\sigma}$ is a functor and a Zariski sheaf.

Proof. The arguments used in the proofs of Proposition 2.3 and Lemma 2.4 also apply to the case of the Gröbner functor.

Theorem 5.3. Let $J$ be an $m$-truncation strongly stable ideal and $\sigma$ a term ordering. Then the Gröbner functor $\underline{\mathbf{S t}}_{J}^{\sigma}$ is a closed subfunctor of $\underline{\mathbf{M f}}_{J}$.

Using Notation 2.5, $\underline{\mathbf{S t}}_{J}^{\sigma}$ is represented by the affine scheme $\mathbf{S t}_{J}^{\sigma}:=$ Spec $\mathbb{Z}[\mathbf{C}] / \mathfrak{I}_{J}^{\sigma}$, where $\mathfrak{I}_{J}^{\sigma}$ is the sum of the ideal $\mathfrak{I}_{J}$ described in Theorem 2.6 and the ideal $\mathfrak{G}_{J}^{\sigma}:=\left(C_{\alpha \beta} \mid x^{\beta}>_{\sigma} x^{\alpha}\right)$.

Proof. The proof is straightforward by applying the criterion given in [16, Proposition 2.9] on the inclusion $\iota: \underline{\mathbf{S t}}_{J}^{\sigma}(A) \hookrightarrow \underline{\mathbf{M f}}_{J}(A)$.

The scheme representing the Gröbner functor is called the Gröbner stratum.

Example 5.4. Let us consider the ideal $J=\left(x_{2}^{2}, x_{2} x_{1}, x_{1}^{3}\right)$ of Example 2.14 and the term ordering DegLex. There is only one monomial in $\mathcal{N}(J)$ greater than some monomial of the same degree in $\mathcal{B}_{J}$ : $x_{2} x_{0}^{2}>_{\text {DegLex }} x_{1}^{3}$. Therefore, the ideal defining $\mathbf{S t}_{J}^{\text {DegLex }}$ as a subscheme of $\mathbb{A}^{12}=\operatorname{Spec} \mathbb{Z}[\mathbf{C}]$ is the sum of the ideal defining $\mathbf{M f}_{J}$ and the principal ideal $\left(C_{030,201}\right)$ and $\mathbf{S t}_{J}^{\text {DegLex }}$ is a hyperplane section of $\mathbf{M f} \mathbf{f}_{J}$.

An analogue of Theorem 3.4 also holds for Gröbner strata (see [22, Theorem 4.7]). In particular, we have an isomorphism $\mathbf{S t}_{J_{\geq s-1}}^{\sigma} \simeq \mathbf{S t}_{J_{\geq s}}^{\sigma}$ under the assumption of Theorem 3.4 (i), leading to the isomorphism 
$\mathbf{M f}_{J_{\geq s-1}} \simeq \mathbf{M f}_{J_{\geq s}}$. From this property, we can deduce some cases in which marked families and Gröbner strata coincide.

We need the following property.

Proposition $\mathbf{5 . 5}$ ([8, Lemma 3.2]). Let $J$ be a saturated strongly stable ideal. If the truncation $J_{\geq m}$ is a gen-segment ideal, then so is $J_{\geq m-1}$. In general, the opposite implication is not true.

Thus, if we consider a strongly stable saturated ideal $J$ without minimal generators divisible by $x_{1}$ in degree $s+1$, then

$$
\mathbf{M f}_{J_{\geq s-1}} \simeq \mathbf{M f}_{J_{\geq s}} \text { and } \mathbf{S t}_{J_{\geq s-1}}^{\sigma} \simeq \mathbf{S t}_{J_{\geq s}}^{\sigma}
$$

If, moreover, we assume that there exists a term ordering $\sigma$ making $J_{\geq s-1}$ a gen-segment ideal, then by Theorem 3.4, we get

$$
\mathbf{S t}_{J_{\geq s}}^{\sigma} \simeq \mathbf{S t}_{J_{\geq s-1}}^{\sigma}=\mathbf{M} \mathbf{f}_{J_{\geq s-1}} \simeq \mathbf{M f}_{J_{\geq s}}
$$

so that $\mathbf{S t}_{J_{\geq s}}^{\sigma}$ and $\mathbf{M f} \mathbf{f}_{J_{>s}}$ coincide, even if $J_{\geq s}$ were not a gen-segment ideal. Note that, in this last case, there exist pairs of monomials $x^{\alpha} \in J_{s}$ and $x^{\beta} \in \mathcal{N}(J) s$ such that $x^{\alpha}<_{\sigma} x^{\beta}$. However, since $\mathfrak{I}_{J_{\geq s}}$ and $\mathfrak{I}_{J_{\geq s}}^{\sigma}$ coincide, the variables $C_{\alpha \beta}$ corresponding to those pairs of monomials must already be contained in $\mathfrak{I}_{J_{\geq s}}$.

Example 5.6. Let us consider the ideal

$$
J=\left(x_{2}^{3}, x_{2}^{2} x_{1}, x_{2} x_{1}^{2}\right) \subseteq \mathbb{Z}\left[x_{0}, x_{1}, x_{2}\right] .
$$

Its Castelnuovo-Mumford regularity is 3 and its Hilbert polynomial is $p(t)=t+4$ with Gotzmann number 4 .

For $s=1,2,3, J_{>s}$ is a gen-segment ideal with respect to any term ordering $\sigma$ induced by a refinement of the grading $(4,3,1)$, whereas $J_{\geq 4}$ cannot be a gen-segment since $x_{2} x_{1}^{2} x_{0} \in J_{4}, x_{1}^{4}, x_{2}^{2} x_{0}^{2} \in \mathcal{N}(J)_{4}$ and $\left(x_{2} x_{1}^{2} x_{0}\right)^{2}=x_{1}^{4} \cdot x_{2}^{2} x_{0}^{2}$. Since there is no minimal generator in degree 5 , the equality $\mathbf{M} \mathbf{f}_{J_{\geq 3}}=\mathbf{S t}_{J_{\geq 3}}^{\sigma}$ induces the equality $\mathbf{M} \mathbf{f}_{J_{\geq 4}}=\mathbf{S t}_{J_{\geq 4}}^{\sigma}$ as subschemes of $\mathbf{H i l b} \mathbf{b}_{2}^{t+4}$, even if our construction defines them in affine spaces of different dimensions.

Indeed, in the construction of $\mathbf{M} \mathbf{f}_{J_{>4}}$ we consider the variable $C_{121,040}$ corresponding to the monomial $x_{1}^{4}$ in the tail of the polynomial $f_{121}$ with $\operatorname{Ht}\left(f_{121}\right)=x_{2} x_{1}^{2} x_{0}$, while this variable does not appear in the 
construction of $\mathbf{S t}_{J_{\geq 4}}^{\sigma}$, since $x_{1}^{4}>_{\sigma} x_{2} x_{1}^{2} x_{0}$. This means that the variable $C_{121,040}$ must already be contained in the ideal defining $\mathbf{M f}_{J_{>4}}$. We will now check this fact, by a direct computation of $\mathfrak{I}_{J_{\geq 4}}$ as in Corollary 2.13.

Among the EK-polynomials involving $f_{121}$ there is

$$
g:=S^{\mathrm{EK}}\left(f_{121}, f_{130}\right)=x_{1} f_{121}-x_{0} f_{130} .
$$

The only monomials in $\operatorname{Supp}(g) \cap J$ are $x_{2}^{2} x_{1} x_{0}^{2}$ and $x_{2} x_{1}^{2} x_{0}^{2}$, both divisible by $x_{0}$. Then

$$
g \stackrel{\mathcal{F}_{J_{\geq 4}}^{(\cdot)}}{\longrightarrow} h=g-\left(C_{121,202}-C_{130,211}\right) \widetilde{f}_{212}-\left(C_{121,112}-C_{130,121}\right) \widetilde{f}_{212},
$$

where

$$
\widetilde{f}_{212}=x_{0} f_{211} \quad \text { and } \quad \widetilde{f}_{122}=x_{0} f_{121} .
$$

Therefore, we replace the monomials $x_{2}^{2} x_{1} x_{0}^{2}$ and $x_{2} x_{1}^{2} x_{0}^{2}$ with linear combinations of monomials all divisible by $x_{0}$, so that the monomial $x_{1}^{5}$ still appears in the support of $h$ with coefficient $C_{121,040}$. Therefore, $C_{121,040}$ is one of the generators of the ideal $\mathfrak{I}_{J_{\geq 4}}$ defining $\mathbf{M f}_{J_{\geq 4}}$.

\section{Example: Marked schemes and Gröbner strata of an ideal} defining seven points in $\mathbb{P}^{3}$. In the final section, we report some results about marked schemes and Gröbner strata associated to the strongly stable ideal

$$
J=\left(x_{3}, x_{2}^{2}, x_{2} x_{1}^{3}, x_{1}^{4}\right) \subseteq k\left[x_{0}, x_{1}, x_{2}, x_{3}\right]
$$

and its truncations. The ideal $J$ defines a point of the Hilbert scheme $\mathbf{H i l b}_{3}^{7}$, which is an irreducible scheme of dimension 21 [23]. As the Gotzmann number is 7 , $\mathbf{H i l b}_{3}^{7}$ can be defined as a subscheme of the Grassmannian $\mathbf{G r}_{7}^{120}$. The Iarrobino-Kleiman equations of the open subscheme $\mathbf{H}_{\mathcal{N}(J)_{7}} \subseteq \mathbf{G}_{\mathcal{N}(J)_{7}}$ can be computed considering the marked scheme $\mathbf{M f}_{J_{\geq 7}}$. By direct computation, one can check that $\mathbf{M f}_{J_{\geq 7}} \simeq \mathbf{H}_{\mathcal{N}(J)_{7}}$ is defined by 2058 quadratic equations in the coordinate ring of the affine space $\mathbb{A}^{791} \simeq \mathbf{G}_{\mathcal{N}(J)_{7}}$. This embedding is clearly inconvenient because of the huge number of variables and the resulting large codimension of $\mathbf{M f}_{J_{\geq 7}}$.

By Theorem 3.4, the marked scheme $\mathbf{M f}_{J_{\geq 7}}$ is isomorphic to the marked scheme $\mathbf{M f}_{J_{\geq 3}}$. The latter scheme is defined as a subscheme 
of $\mathbb{A}^{105}$, its ideal generated by 210 quadratic polynomials, and it turns out to be isomorphic to a rational hypersurface $V_{6}$ in the affine space $\mathbb{A}^{22}$ defined by a degree 6 polynomial. Explicitly finding the embedding Mf $_{J \geq 3} \hookrightarrow \mathbb{A}^{22}$ is the most difficult part of the computation, since the process of elimination of 83 parameters greatly increases the degree of the polynomials. This step can be both time and RAM consuming. We recall that, in order to overcome this difficulty, an alternative polynomial reduction procedure (the so-called superminimal reduction) was developed in [4]. This procedure allows us to embed the marked scheme in an affine space of far lower dimension. For instance, $\mathbf{M f}_{J_{>3}}$ can be embedded in $\mathbb{A}^{28}$. Considering this embedding, we would only need to eliminate 6 parameters (instead of 83).

The superminimal reduction procedure can be seen as a generalization of the procedure used for computing Gröbner strata of zerodimensional ideals in the affine framework. However, we emphasize that the open subscheme $\mathbf{H}_{\mathcal{N}(J)_{7}}$ cannot be studied as a Gröbner stratum. First, the truncation $J_{\geq 3}$ is not a gen-segment ideal. Indeed, the polynomials in the marked basis with head term $x_{2}^{2} x_{0}$ and $x_{1}^{4}$ have respectively $x_{2} x_{1}^{2}$ and $x_{2} x_{1}^{2} x_{0}$ in the support of their tails, but $x_{2} x_{1}^{2}$ and $x_{2} x_{1}^{2} x_{0}$ cannot appear at the same time in generators with initial terms $x_{2}^{2} x_{0}$ and $x_{1}^{4}$ of a reduced Gröbner basis, since we would have

$$
x_{2}^{2} x_{0}>_{\sigma} x_{2} x_{1}^{2}, \quad x_{1}^{4}>_{\sigma} x_{2} x_{1}^{2} x_{0} \quad \text { and } \quad x_{2}^{2} x_{0} \cdot x_{1}^{4}=x_{2} x_{1}^{2} \cdot x_{2} x_{1}^{2} x_{0} .
$$

Second, the coefficients of $x_{2} x_{1}^{2}$ and $x_{2} x_{1}^{2} x_{0}$ in the polynomials of the marked basis with head terms $x_{2}^{2} x_{0}$ and $x_{1}^{4}$ are not contained in the ideal defining $\mathbf{M f} f_{J_{\geq 3}}$ so that the Gröbner stratum $\mathbf{S t}_{J_{\geq 3}}^{\sigma}$ is a proper subscheme for every $\sigma$ (Theorem 5.3). The smallest codimension of a Gröbner stratum contained in $\mathbf{M} \mathbf{f}_{J_{\geq 3}}$ is 1 . In fact, the Gröbner strata corresponding to the term orderings obtained as a refinement of the gradings $(13,6,4,1)$ and $(11,6,3,1)$ are both isomorphic to $\mathbb{A}^{20}$. In the generic Gröbner basis of $\mathbf{S t}_{J_{>3}}^{(13,6,4,1)}$, the monomial $x_{2} x_{1}^{2}$ does not appear in the generator with initial term $x_{2}^{2} x_{0}$, while in the case of $\mathbf{S t}_{J_{\geq 3}}^{(11,6,3,1)}, x_{2} x_{1}^{2} x_{0}$ does not appear in the generator with the initial term $x_{1}^{4}$ (and there are no other differences with the marked basis).

Other proper subschemes of $\mathbf{M f} \mathbf{J}_{J_{3}}$ can be obtained considering marked schemes (and Gröbner strata) of truncation of $J$ in degree $<3$ (the computation in these cases is much simpler and lasts a few 
TABLE 1. Marked schemes and Gröbner strata with respect to the graded lexicographic and reverse lexicographic term orderings of $J, J_{\geq 2}$ and $J_{\geq 3}$, where $J=\left(x_{3}, x_{2}^{2}, x_{2} x_{1}^{3}, x_{1}^{4}\right) \subseteq k\left[x_{0}, x_{1}, x_{2}, x_{3}\right]$.

\begin{tabular}{|c|c|c|c|}
\hline & Marked scheme & $\begin{array}{c}\text { Gröbner stratum } \\
\text { w.r.t. RevLex }\end{array}$ & $\begin{array}{l}\text { Gröbner stratum } \\
\text { w.r.t. DegLex }\end{array}$ \\
\hline$J$ & $\begin{array}{c}\mathbf{M f}_{J} \subseteq \mathbb{A}^{22} \\
28 \text { equations } \\
\mathbf{M f}_{J} \simeq \mathbb{A}^{15}\end{array}$ & $\begin{array}{c}\mathbf{S t}_{J}^{\text {RevLex }} \subseteq \mathbb{A}^{22} \\
28 \text { equations } \\
\mathbf{S t}_{J}^{\text {RevLex }} \simeq \mathbb{A}^{15}\end{array}$ & $\begin{array}{c}\mathbf{S t}_{J}^{\text {DegLex }} \subseteq \mathbb{A}^{19} \\
28 \text { equations } \\
\mathbf{S t}_{J}^{\text {DegLex }} \simeq \mathbb{A}^{12}\end{array}$ \\
\hline$J_{\geqslant 2}$ & $\begin{array}{l}\mathbf{M f}_{J_{\geq 2}} \subseteq \mathbb{A}^{39} \\
77 \text { equations } \\
\mathbf{M f}_{J_{\geq 2}} \simeq \mathbb{A}^{15}\end{array}$ & $\begin{array}{c}\mathbf{S t}_{J_{\geq 2}}^{\text {RevLex }} \subseteq \mathbb{A}^{37} \\
71 \text { equations } \\
\mathbf{S t}_{J_{\geq 2}^{\text {RevLex }}}^{\text {Rev }} \simeq \mathbb{A}^{15}\end{array}$ & $\begin{array}{c}\mathbf{S t}_{J_{\geq 2}}^{\text {DegLex }} \subseteq \mathbb{A}^{36} \\
77 \text { equations } \\
\mathbf{S t}_{J_{\geq 2}^{\text {DegLex }}}^{\text {Dex }} \simeq \mathbb{A}^{12}\end{array}$ \\
\hline$J_{\geqslant 3}$ & $\begin{array}{c}\mathbf{M f}_{J_{\geq 3}} \subseteq \mathbb{A}^{105} \\
210 \text { equations } \\
\mathbf{M f}_{J_{\geq 3}} \simeq V_{6} \subseteq \mathbb{A}^{22} \\
V_{6} \sim-\sim \mathbb{A}^{21}\end{array}$ & $\begin{array}{l}\mathbf{S t}_{J_{\geq 3}}^{\text {RevLex }} \subseteq \mathbb{A}^{93} \\
204 \text { equations } \\
\mathbf{S t}_{J_{\geq 3}^{\text {RevLex }} \simeq \mathbb{A}^{15}}\end{array}$ & $\begin{array}{l}\mathbf{S t}_{J_{\geq 3}^{\text {DegLex }}} \subseteq \mathbb{A}^{102} \\
210 \text { equations } \\
\mathbf{S t}_{J_{\geq 3}^{\text {DegLex }}} \simeq \mathbb{A}^{18}\end{array}$ \\
\hline
\end{tabular}

seconds). In Table 1, we show the comparison between marked schemes and Gröbner strata with respect to the graded lexicographic and reverse lexicographic term orderings of several truncations of $J$. Notice that we already know theoretically that $\mathbf{M f}_{J_{\geq 2}} \simeq \mathbf{M f} \mathbf{f}_{J}$ and $\mathbf{S t}_{J_{\geq 2}}^{\sigma} \simeq \mathbf{S t}_{J}^{\sigma}$, for any $\sigma$. The case of the reverse lexicographic order is even more special, since the Gröbner strata with respect to RevLex of all truncations are isomorphic [22, Proposition 4.11].

Moreover, by direct computation, we observe that the saturated ideal $J$ is a gen-segment with respect to the reverse lexicographic order, so that $\mathbf{M} \mathbf{f}_{J}=\mathbf{S t}_{J}^{\text {RevLex }}$. The truncation $J_{\geq 2}$ is a gen-segment with respect to any term ordering induced by a refinement of the grading $(7,4,3,1)$, so that the marked scheme $\mathbf{M f}_{J_{\geq 2}}$ coincides with the Gröbner stratum $\mathbf{S t}_{J_{\geq 2}}^{(7,4,3,1)}$. Notice that the term ordering induced by $(7,4,3,1)$ allows two monomials more than the reverse lexicographic order in the tails of the marked set. In fact, $\mathbf{S t}_{J_{\geq 2}}^{(7,4,3,1)} \subseteq \mathbb{A}^{39}$ and $\mathbf{S t}_{J_{\geq 2}}^{\text {RevLex }} \subseteq \mathbb{A}^{37}$. However, explicit computation shows that these two monomials cannot appear 
in the tails of a marked basis, since

$$
\mathbf{S t}_{J_{\geq 2}}^{(7,4,3,1)}=\mathbf{M f} \mathbf{f}_{J_{\geq 2}} \simeq \mathbf{M f} \mathbf{f}_{J}=\mathbf{S t}_{J}^{\text {RevLex }} \simeq \mathbf{S t}_{J_{\geq 2}}^{\text {RevLex }}
$$

Acknowledgments. The authors would like to thank Mathias Lederer for his help in strongly improving the first version of this paper.

\section{REFERENCES}

1. Matthias Aschenbrenner, Reduction $\bmod p$ of standard bases, Comm. Alg. 33 (2005), 1635-1661.

2. Edoardo Ballico, Cristina Bertone and Margherita Roggero, The locus of points of the Hilbert scheme with bounded regularity, Comm. Alg. 43 (2015), 29122931.

3. David Bayer, The division algorithm and the Hilbert schemes, $\mathrm{PhD}$ thesis, Harvard University, 1982.

4. Cristina Bertone, Francesca Cioffi, Paolo Lella and Margherita Roggero, Upgraded methods for the effective computation of marked schemes on a strongly stable ideal, J. Symb. Comp. 50 (2013), 263-290.

5. Cristina Bertone, Paolo Lella and Margherita Roggero, A Borel open cover of the Hilbert scheme, J. Symb. Comp. 53 (2013), 119-135.

6. Jerome Brachat, Paolo Lella, Bernard Mourrain and Margherita Roggero, Extensors and the Hilbert scheme, Ann. Sc. Norm. 16 (2016), 65-96.

7. Giuseppa Carrà Ferro, Gröbner bases and Hilbert schemes, I, J. Symb. Comp. 6 (1988), 219-230.

8. Francesca Cioffi, Paolo Lella, Maria Grazia Marinari and Margherita Roggero, Segments and Hilbert schemes of points, Discr. Math. 311 (2011), 2238-2252.

9. Francesca Cioffi and Margherita Roggero, Flat families by strongly stable ideals and a generalization of Gröbner bases, J. Symb. Comp. 46 (2011), 10701084 .

10. David Eisenbud, The geometry of syzygies, Grad. Texts Math. 229, Springer-Verlag, New York, 2005.

11. Shalom Eliahou and Michel Kervaire, Minimal resolutions of some monomial ideals, J. Alg. 129 (1990), 1-25.

12. André Galligo, À propos du théorème de-préparation de Weierstrass, in Fonctions de plusieurs variables complexes, Lect. Notes Math. 409, Springer, Berlin, 1974.

13. Gerd Gotzmann, Eine Bedingung für die Flachheit und das Hilbertpolynom eines graduierten Ringes, Math. Z. 158 (1978), 61-70.

14. Mark L. Green, Generic initial ideals, in Six lectures on commutative algebra, Progr. Math. 166 (1998), 119-186. 
15. Alexander Grothendieck, Techniques de construction et théorèmes d'existence en géométrie algébrique, IV, Les schémas de Hilbert, Sem. Bour. 6 (1995), 249-276.

16. Mark Haiman and Bernd Sturmfels, Multigraded Hilbert schemes, J. Alg. Geom. 13 (2004), 725-769.

17. Robin Hartshorne, Connectedness of the Hilbert scheme, Inst. Haut. Sci. Publ. Math. 29 (1966), 5-48.

18. Anthony Iarrobino and Steven L. Kleiman, The Gotzmann theorems and the Hilbert scheme (Appendix $\mathrm{C}$ of Power sums, Gorenstein algebras, and determinantal loci), Lect. Notes Math. 1721, Springer-Verlag, Berlin, 1999.

19. Mathias Lederer, Gröbner strata in the Hilbert scheme of points, J. Comm. Alg. 3 (2011), 349-404.

20. Paolo Lella, Borel-fixed ideals, available at www.paololella.it/HSC/ Borel-fixed_ideals.html.

21. _ An efficient implementation of the algorithm computing the Borelfixed points of a Hilbert scheme, ISSAC 2012-Proc. 37th Inter. Symp. Symb. Alg. Comp. (2012), 242-248.

22. Paolo Lella and Margherita Roggero, Rational components of Hilbert schemes, Rend. Sem. Mat. Univ. Padova 126 (2011), 11-45.

23. Guerino Mazzola, Generic finite schemes and Hochschild cocycles, Comm. Math. Helv. 55 (1980), 267-293.

24. Roberto Notari and Maria Luisa Spreafico, A stratification of Hilbert schemes by initial ideals and applications, Manuscr. Math. 101 (2000), 429-448.

25. Franz Pauer, On lucky ideals for Gröbner basis computations, J. Symb. Comp. 14 (1992), 471-482.

26. Alyson A. Reeves and Bernd Sturmfels, A note on polynomial reduction, J. Symb. Comp. 16 (1993), 273-277.

27. Stacks Project Authors, The, Stacks project, available at http://stacks. math. columbia.edu.

28. Ravi Vakil, Foundations of algebraic geometry, Stanford University course, Stanford, CA, unpublished notes, 2013.

29. Michael Wibmer, Gröbner bases for families of affine or projective schemes, J. Symb. Comp. 42 (2007), 803-834.

Dipartimento di Matematica, Via Sommarive 14, 38123 Povo Trento, Italy Email address: paolo.lella@unitn.it

Dipartimento di Matematica, Via Carlo Alberto 10, 10123 Torino, Italy Email address: margherita.roggero@unito.it 